\title{
Jean Aurenche, Pierre Bost et Claude Autant-Lara, auteurs de Douce. Genèse d'une pratique scénaristique
}

\author{
Adrien Gaillard et Julien Meyer
}

\section{(2) OpenEdition \\ Journals}

Édition électronique

URL : http://journals.openedition.org/genesis/1533

DOI : 10.4000/genesis.1533

ISSN : 2268-1590

Éditeur :

Presses universitaires de Paris Sorbonne (PUPS), Société internationale de génétique artistique littéraire et scientifique (SIGALES)

Édition imprimée

Date de publication : 27 novembre 2015

Pagination : 91-101

ISBN : 9791023105049

ISSN : 1167-5101

\section{Référence électronique}

Adrien Gaillard et Julien Meyer, « Jean Aurenche, Pierre Bost et Claude Autant-Lara, auteurs de Douce. Genèse d'une pratique scénaristique », Genesis [En ligne], 41 | 2015, mis en ligne le 20 avril 2017, consulté le 17 mai 2019. URL : http://journals.openedition.org/genesis/1533 ; DOI : 10.4000/ genesis. 1533 


\title{
Jean Aurenche, Pierre Bost et Claude Autant-Lara, auteurs de Douce. Genèse d'une pratique scénaristique
}

\author{
Adrien Gaillard et Julien Meyer
}

\section{«Aurenchébost »?}

Les discours portant sur le tandem Jean Aurenche et Pierre Bost se sont déclinés, au fil des décennies, en consécration, déclassement et revalorisation, signalant divers degrés de légitimation au sein du champ cinématographique. Alors que le duo de scénaristes s'imposait dans le second après-guerre comme l'un des plus actifs du cinéma populaire français dit de «la Qualité », le jeune François Truffaut lui asséna un discrédit décisif au cours des années cinquante ; opprobre qui serait en partie levé avec la réhabilitation appelée de ses vœux par Bertrand Tavernier, vingt ans plus tard1. Toujours est-il que l'estampille « Aurenchébost » s'est imposée dans l'imaginaire collectif comme s'il s'agissait d'un seul et même auteur' ${ }^{2}$. Cette perspective moniste offre une commodité rédactionnelle, que ce soit dans la presse populaire ou dans la critique cinéphilique. Elle présente l'avantage de désigner les auteurs de façon univoque et de les ériger en contre-modèles des pionniers de la Nouvelle Vague ${ }^{3}$. Elle sous-tend les discours sur le style d'auteur et rend le travail d'équipe difficile à penser. Érigé en label, le binôme de la « qualité française » constitue par conséquent un phénomène exemplaire pour réfléchir au jeu d'alternance entre les dominants et les dominés du champ ou pour ressaisir une périodisation dans l'histoire du cinéma ${ }^{4}$.

Ces représentations culturelles masquent, dans la caricature obvie des indexations et des comparaisons, ce qui reste des pratiques d'écriture avant tout collectives. La documentation génétique permet d'en observer les traces de façon privilégiée. En effet, là où les discours de réception essentialisent l'activité scénaristique en méthode et en recettes à succès, les archives rendent leur complexité aux productions : il n'est plus question d'en rester à l'écriture idéalisée d'une histoire, mais bien de basculer dans l'his- toricité et la contingence des pratiques d'écriture 5 . C'est dans cette perspective que notre contribution se propose de réfléchir au travail scénaristique d'Aurenche et Bost, un duo auquel il convient d'associer le réalisateur Claude Autant-Lara, puisque ce dernier a bien souvent participé à l'élaboration des scénarios avant de les mettre en scène. Nos analyses se basent d'ailleurs sur le fonds d'archives

1. Voir surtout François Truffaut, «Une certaine tendance du cinéma français », Cahiers du cinéma, n 31, janvier 1954, p. 15-29. Quant à Tavernier, en dehors des films qu'il réalise avec Aurenche et Bost, ou sur eux, il valorise le tandem dans ses entretiens : Jeux d'auteurs, mots d'acteurs : scénaristes et dialoguistes du cinéma français, 1930-1945, Lyon/Arles, Institut Lumière/Actes Sud, 1994, p. 38-50.

2. Une telle amalgamation est courante. Elle est formulée, par exemple, à l'occasion de la remise du prix Louis-Delluc pour L'Horloger de SaintPaul : Jean-Louis Bory, «Vive Aurenchébost ! », Le Nouvel Observateur, 14 janvier 1974.

3. Au moins dès 1955, la « Politique des Auteurs » a dressé progressivement une opposition entre, d'une part, un cinéma dit « traditionnel » étant l'apanage des scénaristes et adaptateurs d'œuvres littéraires, et d'autre part, un cinéma «moderne », celui de la Nouvelle Vague, dont les promoteurs valorisent la mise en scène comme une écriture, comme l'essence de la création cinématographique. François Truffaut, «Ali Baba et la "Politique des Auteurs" », Cahiers du cinéma, n 44, février 1955 , p. 45-47; à ce propos, voir notamment Philippe Mary, La Nouvelle Vague et le cinéma d'auteur: socio-analyse d'une révolution artistique, Paris, Éditions du Seuil, 2006, p. 91-131.

4. Sur ces sujets, voir entre autres : Jean-Pierre Esquenazi, «Arrièregardes et Nouvelle Vague, Le cinéma "qualité France” », dans William Marx (dir.), Les Arrière-gardes au XXe siècle. L'autre face de la modernité esthétique, Paris, PUF, 2004, p. 69-78 ; Laurent Le Forestier, Guillaume Vernet, « La "Qualité française”" contribution à l'archéologie d'un canon critique », Pietro Bianchi et alii (dir.), Il canon cinematografico/The film canon, Udine, Università degli Studi di Udine, 2011, p. 411-417.

5. Jean Aurenche rejettera d'ailleurs l'idée d'une « méthode » : «En fait, on n'avait pas de méthode, ou plutôt on en avait cent qui variaient selon le lieu et l'heure et des tas d'impondérables. C'est difficile à expliquer », Jean Aurenche, La Suite à l'écran, entretiens avec Anne et Alain Riou, Lyon/Arles, Institut Lumière/Actes Sud, 2002 [1993], p. 90-91. 
du réalisateur, dont la Cinémathèque suisse de Lausanne est dépositaire. Elles se focaliseront sur la genèse du film Douce (sorti dans les salles en 1943), qui réunissait pour la première fois le tandem et Autant-Lara6.

\section{Formation d'un trio d'auteurs}

En octobre 1942, peu de temps après leur collaboration sur Le Mariage de Chiffon, Aurenche et Autant-Lara signent pour Douce, adaptation d'un roman publié un an auparavant par Hélène Marty sous le pseudonyme de Michel Davet. Le choix du sujet revient à $\mathrm{Au}-$ renche, qui compte déjà une vingtaine de scénarios à son actif. C'est sur le conseil d'une amie qu'il aurait découvert, puis apprécié le roman. L'action fait écho aux souvenirs de sa mère, autrefois gouvernante dans une riche famille de Lyon. Pour Aurenche, l'investissement personnel dans la lecture est synonyme d'adaptation : « Tout lecteur est un adaptateur qui lit avec ses propres lunettes7. » Mais cette appropriation commence surtout par une reconfiguration dramatique qui induit, entre autres, la transposition du narrateur (Marie-Claire) en personnage (rebaptisé Irène). Elle passe également par des « greffes » effectuées à partir du roman, comme la réplique «Vous voulez savoir si nous couchons dans le même lit 8 ? » insérée littéralement dans le synopsis ${ }^{9}$. Par ailleurs, ce dernier ne se limite pas à la rédaction d'un argument dramatique. Au verso de la page 4 sont inscrits de la main d'Aurenche un calcul prévisionnel du métrage, subdivisé selon la tripartition aristotélicienne, ainsi qu'une liste de vedettes (fig. 1). Si Aurenche semble être l'initiateur d'un premier travail d'adaptation, le projet prend d'emblée une dimension collective.

Douce séduit une société de production, l'Industrie cinématographique, qui fixe le tournage au début de l'année suivante. D'après les contrats, Aurenche est chargé de l'adaptation et des dialogues, Autant-Lara de la réalisation - ce qui comprend le travail du découpage technique 10. Dans les faits, ils développent ensemble le scénario, des semaines durant, comme le laisse supposer une lettre qu'ils adressent à la vedette Marguerite Moreno, alors pressentie pour jouer le rôle de la comtesse de Bonafé11. Mais cette entreprise va rapidement s'enrayer. Accusant un retard sur l'échéancier dès janvier 1943, Aurenche abandonne l'écri-

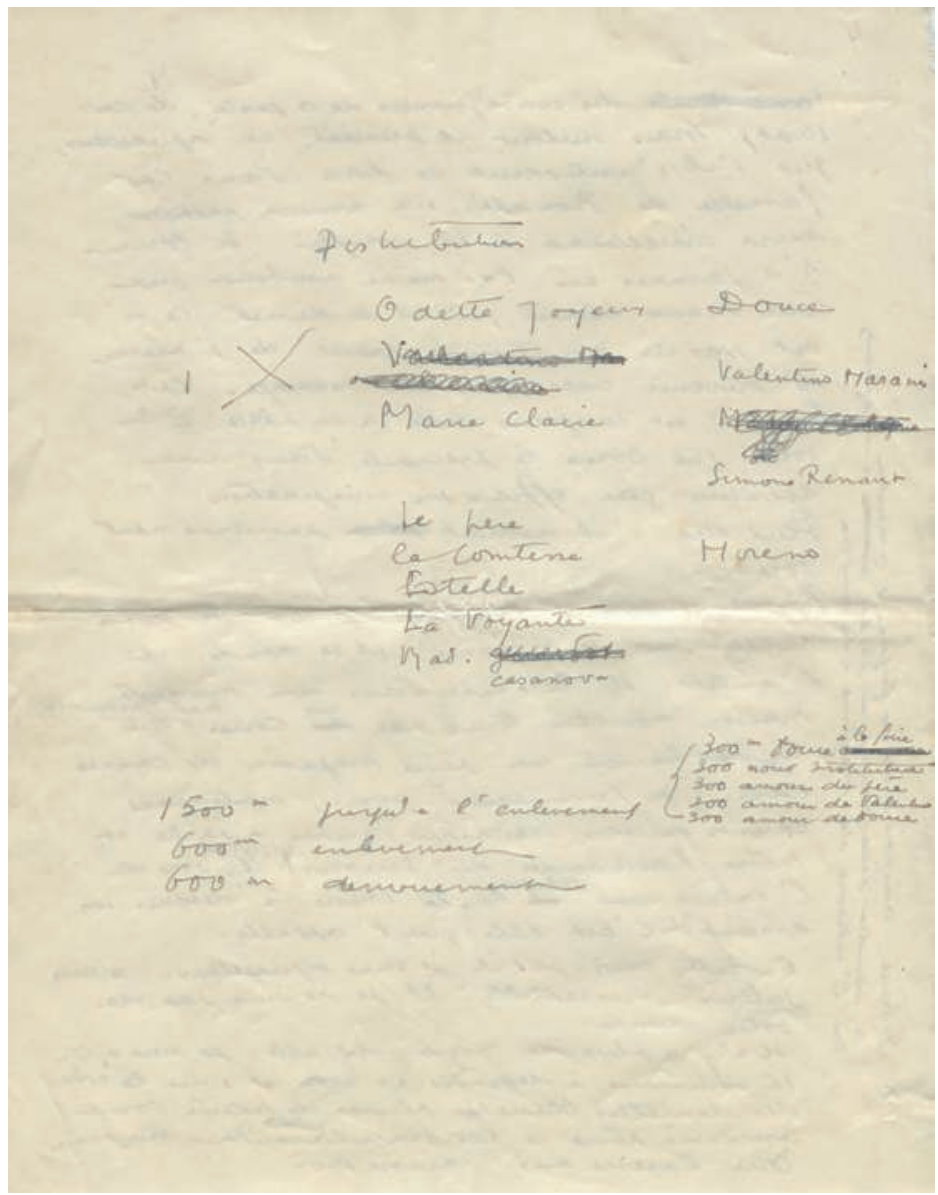

Fig. 1 : Extrait de notes de la main de Jean Aurenche, verso de la quatrième page du synopsis de Douce, 1942.

Fonds Claude Autant-Lara (CSL.5), 95/7 A4.1, Collection Cinémathèque suisse

6. La présente étude s’inscrit dans le projet « Discours du scénario : étude historique et génétique des adaptations cinématographiques de Stendhal » financé par le Fonds national suisse de la recherche scientifique (FNS 100013_149394/1) et mené dans le cadre de la collaboration UNIL et Cinémathèque suisse. Elle fait écho à une hypothèse méthodologique avancée par Alain Boillat, « À la recherche du scénario. Le scénario comme objet (d'étude) et pratique (d'écriture) », Archipel, n 34, 2011, p. 13-59. Les cotes citées, sauf mention contraire, renvoient au Fonds Claude Autant-Lara, Cinémathèque suisse de Lausanne (CSL.5).

7. La Suite à l'écran, op. cit., p. 125, p. 237. Aurenche file la métaphore de l'emménagement pour évoquer son travail : « [...] quand on y met beaucoup de soi-même, on dérange en s'installant. [...] Quelquefois, on s'installe trop et on casse les meubles », p. 121-122.

8. Michel Davet, Douce, Paris, Flammarion, coll. « J'ai lu », 1966 [1940], p. 153.

9. Ce document de la plume d'Aurenche (95/7 A4.1) est intitulé « Résumé de Douce » et date de novembre 1942. Il comporte cinq feuillets paginés recto verso avec peu de ratures ; principalement des variantes immédiates d'écriture. À sa manière, il témoigne des vestiges du passage de l'exogenèse à l'endogenèse, bien décrit par Debray Genette à propos de Flaubert : « [...] transformation verbale, enchâssement, effacement des signes d'intertextualité, tout cela forme le travail de l'écriture, qui s'intériorise peu à peu », Raymonde Debray Genette, « Génétique et théories littéraires », mis en ligne le 15 décembre 2007 : <www.item.ens.fr/index.php?id=200864>.

10. Contrats du 16 octobre 1942, 94/1 A 2.1 .

11. Lettre du 25 octobre $1942,94 / 5$ A3. 
ture des dialogues pour s'en tenir à la fonction d'adaptateur. La charge de dialoguiste sera attribuée temporairement à Steve Passeur, dramaturge et scénariste populaire qui avait travaillé avec Abel Gance et Marcel L'Herbier12.

La production prend encore du retard pour des raisons administratives. Le gouvernement de Vichy ne délivre pas le laissez-passer nécessaire à la participation de Marguerite Moreno13. C'est à la faveur de ce contretemps que Pierre Bost est engagé pour dialoguer le film, probablement à la demande d'Aurenche. Ce dernier n'apprécie guère Passeur, qu'il associera dans ses entretiens au mauvais théâtre de l'époque ${ }^{14}$. De plus, il garde un bon souvenir de Bost, libéré depuis deux ans des geôles allemandes ${ }^{15}$ et avec lequel il a travaillé par téléphone sur L'Héritier des Mondésir d'Albert Valentin en 1935. Les deux auteurs s'étaient alors découvert une certaine affinité au travail. Aurenche admire chez son partenaire, encore débutant dans le cinéma, la carrure d'un homme de lettres accompli, qui cumule les expériences de critique, romancier, dramaturge et demeure une figure intégrée dans le cercle de la Nouvelle Revue française. Enfin, au moment où Aurenche « sèche » sur la rédaction des « trente ou quarante premières pages » de Douce ${ }^{16}$, Bost est sollicité pour l'efficacité rédactionnelle qu'on lui prête, notamment en ce qui concerne les dialogues ${ }^{17}$.

La genèse scénaristique se poursuit en trio, non sans quelques dissensions au terme du projet. Odette Joyeux (qui incarne Douce) relate deux fins alternatives qui auraient fait l'objet d'un débat. À la différence de la version défendue par Aurenche et Bost, empreinte d'émotion romanesque, Autant-Lara a choisi de privilégier un dénouement scellant les clivages sociaux, sans doute dans le but de " "lapider" la bonne bourgeoisie18 »: Irène, l'institutrice, et Fabien, l'intendant de la maison, sont congédiés par la comtesse de Bonafé à la suite du décès de Douce. «On songe à Maupassant, un Maupassant qui aurait lu Marx », écrira Pierre Marcabru dans Arts en 196019. Malgré l'inactualité du sujet (de mise durant l'Occupation), situant l'action en 1887 , le film a fait les frais de la censure vichyste pour ses sous-entendus politiques, en particulier à cause de la scène de la visite aux pauvres ${ }^{20}$. Cette censure couvait déjà tout au long de l'écriture du film, puisque Jean Debucourt, sociétaire de la Comédie-Française et interprète du comte de Bonafé, était chargé d'influencer le découpage scénaristique pour le compte de Vichy 21 .

\section{Vision et division du travail}

La genèse de Douce nous laisse entrevoir les aléas d'une planification, tributaire de concours de circonstances et de contraintes aussi personnelles que collectives. Avant d'en passer à l'analyse des pratiques d'écriture en soi, il convient ainsi de nous prémunir contre deux modes d'appréhension du travail scénaristique. Le premier, d'allure synthétique, conçoit l'activité collective dans sa dimension unitaire et consensuelle. Cette approche fut celle de François Truffaut pour qui le duo Aurenche et Bost constituait les rouages complémentaires d'un «système », avec Bost en « technicien » et Aurenche comme « part spirituelle de la commune besogne 22 ». La description de Truffaut laisse transparaître en filigrane l'ombre portée d'une seule autorité créatrice, résumable à une intention et fournissant, accessoirement, une seule et même

12. Lettre de Claude Autant-Lara à Pierre Guerlais (producteur délégué), le 5 janvier 1943 (94/5 A3).

13. Les sources sont lacunaires à ce sujet. On sait cependant que le tournage, d'abord déplacé en mars, n'est toujours pas d'actualité en mai. Voir les six cartes postales de Marguerite Moreno à Claude Autant-Lara, entre décembre 1942 et mai 1943 (94/5 A3).

14. La Suite à l'écran, op. cit., p. 72, 157, 177.

15. Pierre Bost, Un an dans un tiroir, Paris, Le Dilettante, 2010, p. 7-13.

16. La Suite à l'écran, op. cit., p. 125.

17. Ils ont fait sa réputation ; par exemple ceux de Pierre Sylvanès et Simon Joyeuse, personnages du roman Le Scandale, Paris, Gallimard, 1931. À ce propos : François Ouellet, « Pierre Bost », Nuit blanche, magazine littéraire, $\mathrm{n}^{\circ}$ 93, 2003-2004, p. 40-43.

18. Propos d'Odette Joyeux, recueillis en mai 1990, dans Christian Gilles, Le Cinéma des années quarante par ceux qui l'ont fait, t. III, Le cinéma de l'Occupation : 1940-1944, Paris, L'Harmattan, coll. " Champs visuels », 2000, p. 115-116.

19. Cité dans Freddy Buache, Claude Autant-Lara, Lausanne, L’Âge d'Homme, 1982, p. 36

20. C'est la réplique de Fabien à propos d'Irène qui posa problème «Souhaitez-lui l'impatience et la révolte ! »- écrite de la main d'Aurenche, puis reprise sous la plume d'Autant-Lara (94/11 A4.1).

21. Lettre de R. Rian, du Cabinet civil du chef de l'État de Vichy, à Jean Debucourt, 9 octobre 1943 (94/5 A3).

22. Ensemble, ils sont taxés d'«alibisme » pour avoir exalté des idées antibourgeoises et anticléricales, sous couvert d'adaptations d'œuvres littéraires ; Truffaut, art. cité, 1954, p. 16-17, p. 19-20. 
cible dans un argumentaire à charge ${ }^{23}$. A contrario, les points de vue rétrospectifs des différents collaborateurs sur un même film montrent qu'ils ne partagent pas forcément la même vision de ce qu'ils ont produit 24 . Tout au plus avons-nous affaire à des visées communes circonstanciées, comme lorsqu'Autant-Lara, Aurenche et Bost cosigneront une lettre en 1944 pour demander au Comité de libération du cinéma de rétablir la scène des pauvres dans Douce : « [...] le désir des trois auteurs de ce film est que cette scène (toute tournée, toute montée) soit remise dans le film pour qu'il reprenne sans déformation, son sens premier ${ }^{25}$. »

Un second cliché porte sur une division absolue du travail. Il mobilise un imaginaire industriel, nourri par les crédits des génériques et par les contrats de production. Cette perspective trouve son expression paroxystique dans les superproductions hollywoodiennes, qui vont jusqu'à la mise en concurrence de plusieurs auteurs sur un même projet $^{26}$. À l'image du film de Jacques Tati, Jour de fête (1949), dans lequel le personnage du facteur se retrouve en décalage avec le modèle d'une productivité à l'américaine, le cinéma français des années quarante et cinquante ne semble correspondre que très lointainement à la rationalisation hollywoodienne. En matière d'écriture scénaristique, une répartition officielle des tâches (adaptation et dialogues) constitue la surface administrative d'un ensemble d'arrangements tacites favorisant une organisation souple et remaniable. Les contrats d'engagement fixent les sujets et les délais, mais ils réservent bien souvent la possibilité à un auteur de s'entourer d'un collaborateur selon ses affinités ou de sous-traiter : le choix d'Aurenche porté sur Bost, tout comme le retrait de Passeur, vont dans ce sens. Dans le même ordre d'idées, Aurenche a souvent relevé l'absurdité de dissocier les dialogues du reste du travail scénaristique, en reprenant la formule de Jacques Prévert : "C'était comme si on demandait à des gens [...] de ne s'occuper dans un tableau que des arbres, en ignorant totalement le reste de la toile27. » L'attribution courante des dialogues à Bost et de l'adaptation à Aurenche est ainsi simplificatrice, voire erronée.

De fait, les présupposés d'une vision ou d'une division du travail ne permettent de décrire la pratique scénaristique qu'en renforçant au passage un clivage entre deux logiques pourtant consubstantielles : les choix artistiques et les contraintes économiques ${ }^{28}$. Elles représentent la collaboration à partir d'idéaux de réception ou de production qui ne rendent pas vraiment compte de l'objet scénario ni des contingences liées à son écriture.

\section{Continuités dialoguées, découpages techniques}

Les documents scénaristiques appellent deux niveaux d'analyse en regroupant des dimensions avant-textuelle et textuelle. D'une part, ils sont considérés comme des objets transitifs, inscrits dans une chronologie bornée par le contrat d'écriture et le produit filmique définitif ${ }^{29}$. Ils

23. Même dans sa critique positive de La Traversée de Paris, lorsqu'il cite plus exhaustivement les différents collaborateurs/auteurs du film (Autant-Lara et Marcel Aymé), Truffaut parle de « fusion de quatre personnalités », en ne considérant comme réussi que ce qui fait consensus et anéantit les disparités ; François Truffaut, Les Films de ma vie, Paris, Flammarion, 1975, p. 190.

24. La conclusion préalable de Howard Becker sur « un sentiment commun de la valeur » d'une production collective (Les Mondes de l'art, trad. Jeanne Bouniort, Paris, Flammarion, 2010 [1982], p. 63) paraît discutable en ce qui concerne l'écriture de scénarios. Pour exemple, Aurenche reniera la scène de l'hôtel dans La Traversée de Paris, au point d'exiger sa suppression, sans succès face à la désapprobation de ses collaborateurs (195/7 A3)

25. Lettre de Pierre Bost, Claude Autant-Lara et Jean Aurenche à Louis Daquin, le 3 novembre 1944 (94/5 A3).

26. Régine Hollander, «Moule, meule et laminoir : du film hollywoodien contemporain en tant que produit industriel », Genesis, $\mathrm{n}^{\circ} 28$, « Cinéma », 2007 , p. 33.

27. La Suite à l'écran, op. cit., p. 92. À propos de la formule de Prévert, voir Carole Aurouet, « Du visuel au verbal : la méthode d'écriture scénaristique de Jacques Prévert. L'exemple des Visiteurs du soir », Genesis, n²8, « Cinéma », 2007, p. 133.

28. Laurent Creton, Économie du cinéma. Perspectives stratégiques, Paris, Armand Colin, 2009, p. 14 ; voir aussi Joël Augros, « Du scénario comme entité économique ou cette scène vaut cinq millions », dans Territoires du scénario, dir. René Monnier et Anne Roche, Dijon, Centre Gaston Bachelard, 2006, p. 231.

29. Comme le souligne Pier Paolo Pasolini, le scénario est sous-tendu par un inachèvement (« la volonté d'une forme »), puisqu'il tend vers l'objet filmique, sans l'atteindre d'un point de vue ontologique ; Pier Paolo Pasolini, « Le scénario comme structure tendant à être une autre structure », dans L'Expérience hérétique : langue et cinéma, Paris, Payot, 1976, p. 157. Les processus scénaristiques n'étant pas destinés a priori à la publication, il est difficile de concevoir et d'établir leur orientation téléologique en fonction d'un texte « définitif ». 
témoignent d'un processus scriptural caractérisé par des éléments de raturage et de réécriture ${ }^{30}$. Ces éléments renvoient bien souvent à des phases de travail en solitaire, que l'on pourrait qualifier d'intralocutives, moins conservées dans les archives de scénarios. Il en existe néanmoins un bel exemple avec les premiers brouillons d'Aurenche, peu avant la participation de Bost : six feuillets recto verso regroupant les ébauches du dialogue inaugural de Douce. Le scripteur y bute sur des amorces, en multipliant de nombreuses variantes immédiates : il biffe, reprend à la ligne ou sur une nouvelle page (fig. 6) ${ }^{31}$. D'autre part, la scénarisation est rythmée par des finalités intermédiaires. Les documents sont produits dans un cadre collectif avec des tours de lecture et d'écriture ${ }^{32}$. Par conséquent, un destinataire susceptible de lire, d'écrire et de corriger implique des achèvements discursifs et l'attente de « formes types de structuration du genre 33 ». Cette dimension interlocutive permet d'expliquer, dans la plupart des documents scénaristiques, un souci de lisibilité et des formats codifiés - la continuité dialoguée et le découpage technique.

Le fonds Autant-Lara conserve ces différents types de documents retraçant la genèse de Douce dans l'éparpillement des différentes strates. À défaut d'être à la pièce, l'état d'inventaire les regroupe par scènes ou tranches de films. En premier lieu sont archivées une continuité de la plume d'Aurenche pour le début du film (sans doute la version de décembre 1942), ainsi que plusieurs continuités dactylographiées aux encres bleue, noire et violette dans des états fragmentaires. Celles-ci sont annotées et raturées par les trois auteurs; la trace de la plume de Bost nous indique qu'elles ont été produites en 1943. En second lieu, on trouve de nombreuses bribes de découpages techniques, encore plus incomplètes, qui rendent la reconstitution d'une chronologie génétique périlleuse. Selon les scènes, ces découpages se déclinent en plusieurs copies dactylographiées, annotées par différents scripteurs. Quelquefois, ils sont entièrement de la main d'Autant-Lara, paginés ou non, inscrits au verso d'un découpage dactylographié antérieur ${ }^{34}$. À tout cela s'ajoutent plusieurs feuillets volants qui sont insérés dans des séries continues ${ }^{35}$. Dans le cadre de cette contribution, nous ne produirons pas la chronologie complète de Douce, mais nous nous restreindrons à quelques remarques sur des caractéristiques de forma- tage et de pagination dans la mesure où celles-ci peuvent établir des références communes pour les scénaristes et favoriser ainsi un travail à plusieurs.

Rappelons d'abord que la continuité dialoguée et le découpage technique sont interdépendants. Dans la première phase du chantier, en décembre 1942, Autant-Lara est censé découper au fur et à mesure ce qu'Aurenche dialogue, avec un suivi hebdomadaire de la production ${ }^{36}$. Cette cadence de travail impose un formatage typographique et textuel qui rend solidaires les deux étapes en fonction d'une structuration latente du film. Dès les premières ébauches rédactionnelles, Aurenche écrit les didascalies à gauche et les répliques à droite ${ }^{37}$. Ce préformatage du découpage est également manifeste dans le travail de textualisation,

30. Pour Jean-Louis Lebrave, il s'agit de « substitutions chronologiquement orientées », traces d'une « double locution » où se combinent l'activité du scripteur et celle du lecteur ; Jean-Louis Lebrave, « Lecture et analyse du brouillon », Langages, ${ }^{\circ} 69,1983$, p. 11-23 ; Le Jeu de l'énonciation en allemand d'après les variantes manuscrites des brouillons de H. Heine, thèse sous la direction de M. Paul Valentin, Université de Paris-Sorbonne, 1987. 31. 94/9 A4.1.

32. Daniel Ferrer parle d'une externalisation de la «polémique créatrice », au sens du dialogisme bakhtinien, ayant cours dans la genèse collaborative du cinéma ; Daniel Ferrer, Logiques du brouillon. Modèles pour une critique génétique, Paris, Éditions du Seuil, coll. « Poétique », 2011, p. 128.

33. Cette formule de Bakhtine (Esthétique de la création verbale, Paris, Gallimard, 1984 [1952-1953], p. 283) est mobilisée par Rudolf Mahrer et Valentine Nicollier Saraillon dans la distinction qu'ils opèrent entre la notion d'achèvement en critique génétique et en linguistique textuelle. Leur réflexion sur la dialectique avant-texte/texte s'avère particulièrement riche pour des études scénaristiques. «Les brouillons font-ils texte ? Le cas des plans prérédactionnels de C.F. Ramuz », dans Jean-Michel Adam (dir.), Faire texte. Frontières textuelles et opérations de textualisation, Besançon, Presses universitaires de Franche-Comté, 2015, p. 195-275.

34. Le fonds conserve tout de même le manuscrit relié d'un découpage technique complet, dont l'état et la teneur des notes ne laissent aucun doute sur la fonction de support pour le tournage et le montage - d'où cet ajout cocasse à côté d'une indication sonore de bruit de porte, p. 64 : «Oublié merde ! / manque au film !! » (95/8 A4.1).

35. La contribution manuscrite de Bost très localisée (en dehors de corrections que l'on observe çà et là) relève presque exclusivement de ce type, attestant une participation dans un second temps.

36. Lettre de Claude Autant-Lara à Pierre Guerlais, le 1er décembre 1942 (94/5 A3).

37. 94/9 A4.1, CSL.5. La séparation typographique semble être un standard de l'écriture scénaristique depuis le parlant; Isabelle Raynauld, Lire et écrire un scénario. Le scénario de film comme texte, Paris, Armand Colin, 2012, p. 34. 


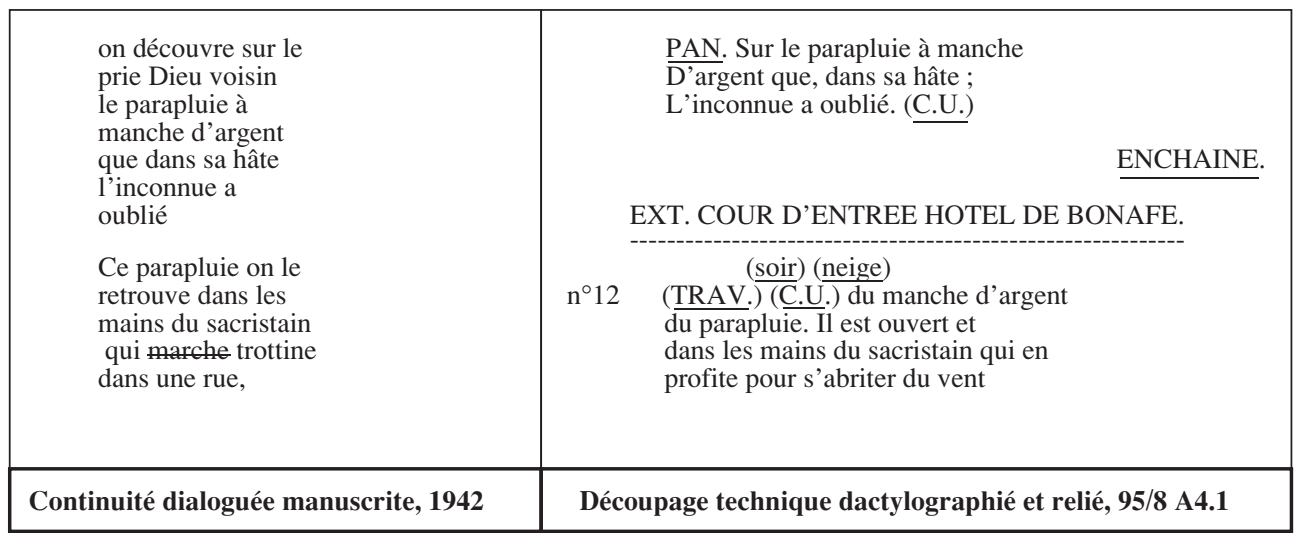

comme en témoigne la page 9 de la continuité d'Aurenche (94/9 A4.1) (voir ci-dessus). Le passage à la ligne suggère l'enchaînement cinématographique, tandis que la dislocation à gauche 38 (« Ce parapluie on le retrouve »), en thématisant l'objet perçu, évoque le procédé du close-up $\left(«\right.$ C.U. ») ${ }^{39}$.

Si les formes de la continuité préparent le découpage, ces opérations sont aussi largement réversibles. Aurenche et Bost réécrivent en fonction ou à la suite des découpages. Dans certains cas, ils interviennent directement sur ceux-ci au même titre qu'Autant-Lara corrige leurs continuités. En témoigne ce feuillet (fig. 2) qui condense le dactylogramme d'un découpage (étape 1), sur lequel Aurenche apporte des corrections manuscrites (étape 2), et dont le pan inférieur a été recouvert par le collage d'un manuscrit de Bost (étape 3), avant qu'Autant-Lara ne lui ajoute de sa plume les indications scéniques « $\underline{\mathrm{Ch}}$ [ambre]. Douce. (jour) » (étape 4). Un tel feuillet rend compte de l'aboutissement complexe d'une suite d'opérations qui ne sont pas forcément enchaînées causalement : mise au propre, lecture corrective, rédaction annexe, substitution par collage et assemblage. À noter que le découpage technique laisse place aux réajustements, puisque le système de numérotation des plans, dans la colonne tout à gauche, attend d'être complété (« $\left.\mathrm{N}^{\circ} »\right)$. Il arrive même que le découpage indique un certain nombre de plans à réserver pour une scène donnée et les résume sous la forme d'une consigne rédactionnelle (fig. 3). Le découpage technique d'Autant-Lara se fait donc en parallèle de la continuité dialoguée d'Aurenche et Bost, dans un aller-retour entre des processus d'écritures dramatique et technique ; ce qui explique le résultat final de certains manuscrits du trio, particulièrement «écrits » et volumineux.

Par ailleurs, le chantier scénaristique mobilise plusieurs systèmes de pagination (fig. 4). Soit ceux-ci fonctionnent de façon conjonctive en unifiant, dans les en- sembles de la continuité ou du découpage, des éléments parfois hétérogènes (brouillon manuscrit, scène dactylographiée, etc.) ; il s'agit alors de tendre vers l'unité finale du scénario. Soit ils fonctionnent de façon disjonctive avec l'introduction d'une pagination autonome qui délimite une scène destinée à être glissée dans l'état actuel de la continuité. L'insertion de nouveaux systèmes facilite la distinction de véritables cellules dramatiques, unités matérielles supérieures à la page, qui font l'objet d'un travail à part. Pour exemple, les auteurs ont choisi d'intercaler une courte scène dans un état avancé du découpage technique entre l'épisode de la fugue des amants (Fabien et Douce) et celui de l'irruption d'Irène dans leur chambre d'hôtel. Cette scénette permet de camper l'inquiétude ambiante à l'hôtel Bonafé et de motiver la venue d'Irène. L'insert est d'abord tapé sous forme de continuité et paginé de 1 à 3, corrigé de la main d'Aurenche et de Bost, recopié de façon manuscrite par Autant-Lara avec des indications supplémentaires (paginé de $\mathrm{A}$ à D), puis tapé à la machine pour être intégré dans la série du découpage préexistant entre la page 11A (au bas de laquelle Aurenche ajoutera l'annotation «fermeture fondu ») et $12 \mathrm{~A}^{40}$. Les multiples systèmes de pagination rendent possibles les ajouts ponctuels, les suppressions et les déplacements des inserts. Dans le cadre d'une genèse en collaboration, ils concilient la souplesse nécessaire à toute création dramatique et la garantie d'une lisibilité, voire d'une supervision partagée par chacun. De ce point de vue, le recours fréquent aux paginations fait écho à l'obsession d'une mise au propre

38. D’un point de vue syntaxique, le complément d'objet direct (« parapluie ») est détaché en tête de phrase pour travailler un effet de focalisation.

39. Autant-Lara semble utiliser dans ses découpages les abréviations d'un jargon technique américain, habitude sans doute due à son passage à Hollywood au début des années trente.

40. 95/1 A4.1. 


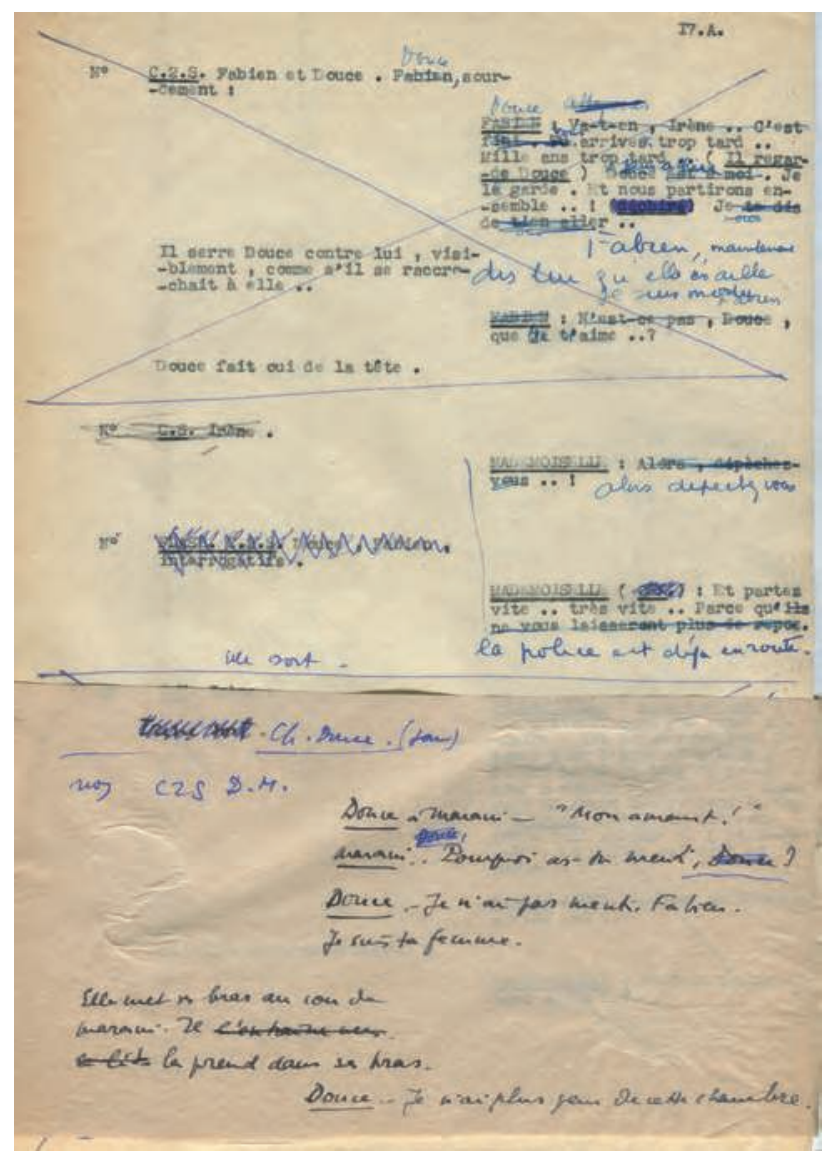

Fig. 2 : Extrait d'un découpage dactylographié de Douce, 1943, raturé et annoté par Jean Aurenche avec ajout par collage d'une continuité dialoguée de la main de Pierre Bost, raturée et annotée par la main de Claude Autant-Lara.

Fonds Claude Autant-Lara (CSL.5), 95/1 A4.1, Collection Cinémathèque suisse

dactylographiée, procédé manifeste dans l'ensemble des documents à tous les stades de la genèse. Il ne faut pas en conclure à un achèvement à un niveau génétique (si ce n'est dans le sens d'une étape intermédiaire), mais bien à un niveau pragmatique, puisqu'il s'agit à tout moment de répondre à l'exigence de donner à lire ou de poursuivre le travail sur la base d'un état validé en commun.

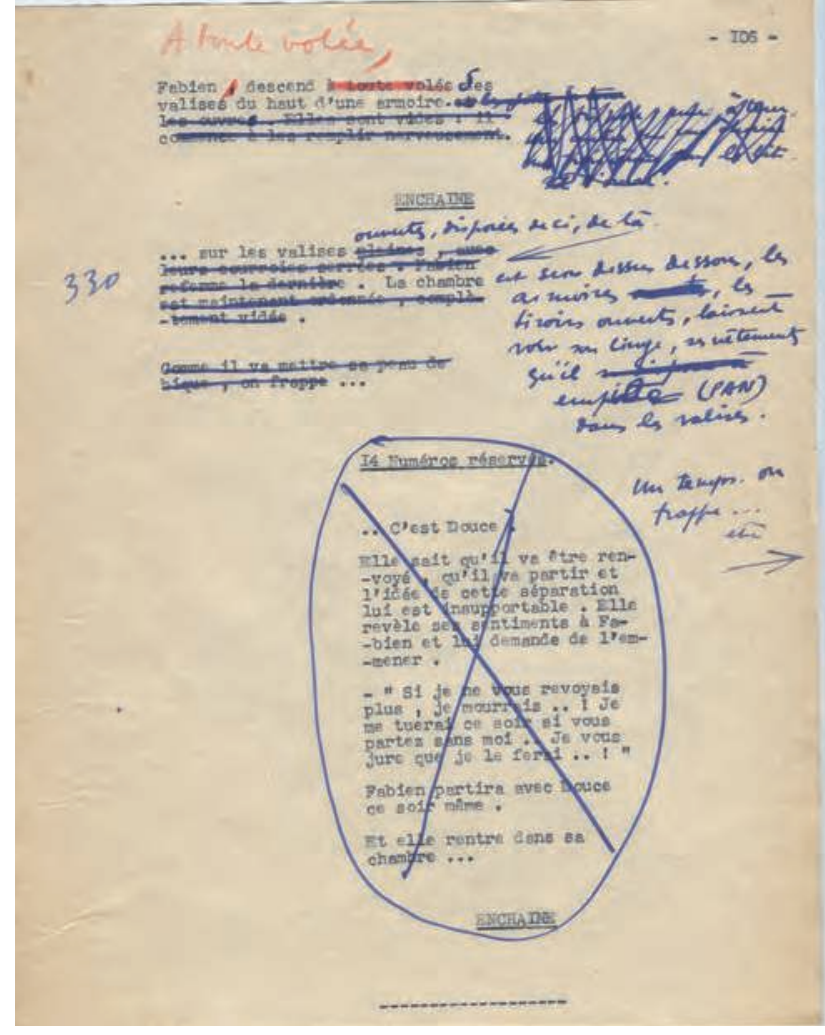

Fig. 3 : Extrait d'un découpage technique dactylographié de Douce, 1943, avec ratures et annotations de la main de Claude Autant-Lara. Fonds Claude Autant-Lara (CSL.5), 94/13 A4.1, Collection Cinémathèque suisse

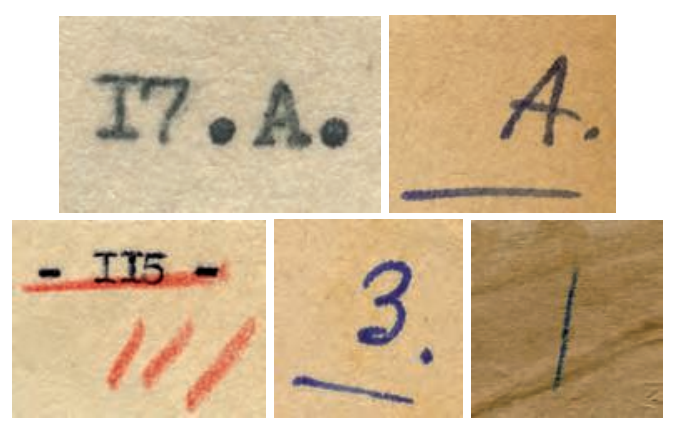

Fig. 4 : Détails de différents systèmes de pagination de continuités dialoguées et de découpages techniques de Douce, 1943. Fonds Claude Autant-Lara (CSL.5), 95/1 A4.1, Collection Cinémathèque suisse 


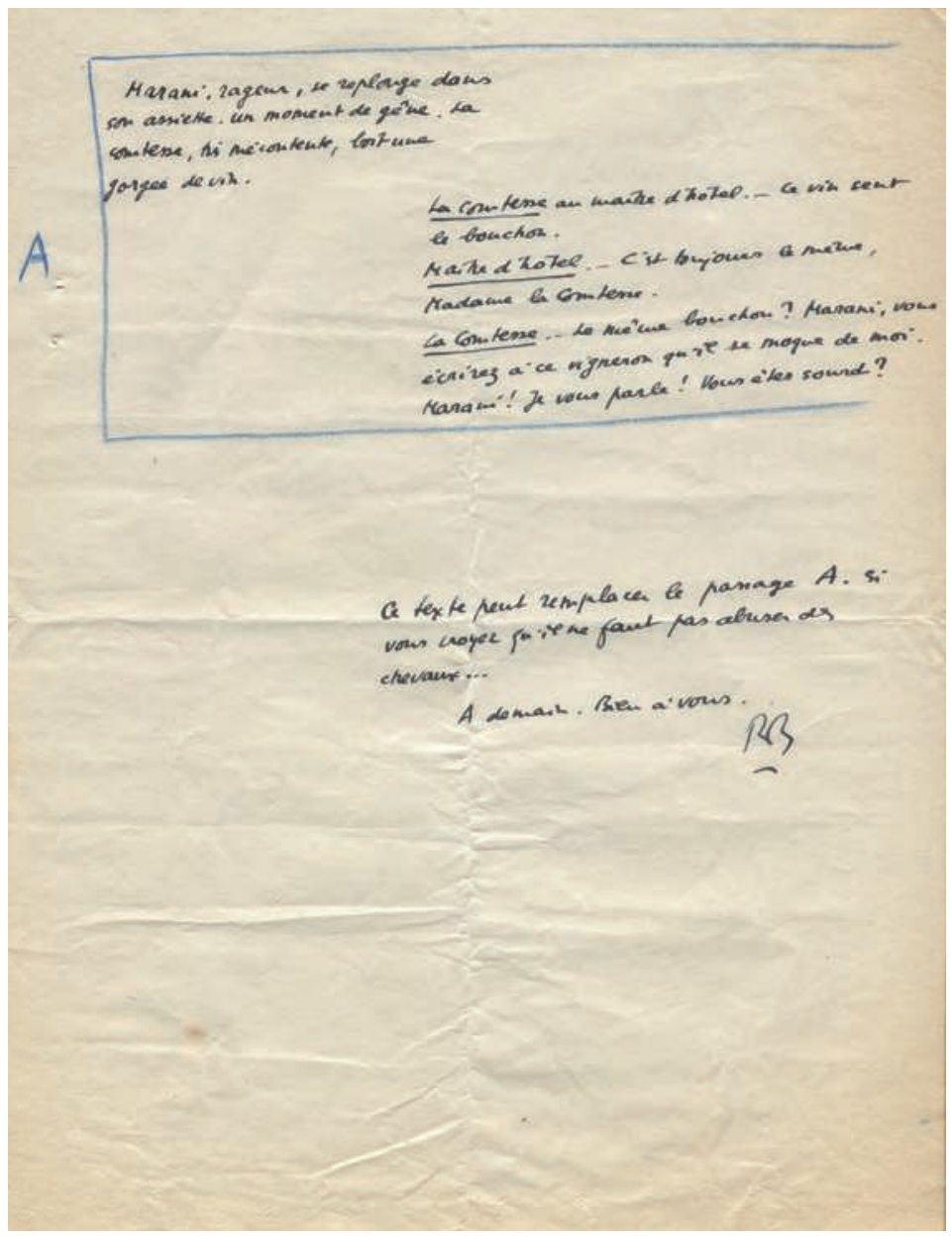

Fig. 5 : Extrait d'une continuité dialoguée de la main de Pierre Bost, 1943. Conservé dans le fonds Claude Autant-Lara (CSL.5), 94/13 A4.1, Collection Cinémathèque suisse

En définitive, la pagination et le formatage s'avèrent indispensables à l'établissement du cadre interlocutif et, par là, à un avancement concerté du projet. Ils participent d'une pratique de manipulation matérielle (analogue au montage filmique) pour faire face à l'ensemble des bifurcations possibles auxquelles est astreinte toute création ${ }^{41}$. Ces bifurcations sont négociées au terme des tours d'écriture ou de lecture. À ces moments clés, les collaborateurs formulent des remarques métatextuelles. Particulièrement instructives en ce qui concerne le travail collectif, elles appa- raissent sous forme de commentaires en marge des feuillets $^{42}$. Autant-Lara est coutumier de ce type de notes qu'il inscrit au crayon noir. Elles portent sur des critères formels - « trop long »/《 plus long »-, ou dramatiques - « on ne sent pas que son rêve est brisé43 ». De façon moins lapidaire, Bost propose des solutions de rechange pour des scènes en laissant le soin à ses partenaires d'en délibérer (fig. 5). Si ce métatexte fournit des informations génétiques de premier ordre, il constitue une part négligeable par rapport à un ensemble de processus interlocutifs éphémères. Il convient alors de distinguer les opérations à distance (sous la forme de tours d'écriture) et les opérations de proximité qui se font à l'oral (conversations rédactionnelles sous la forme de tours de parole), sur lesquelles nous terminons ce modeste passage en revue.

\section{L'auctorialité à l'épreuve des conversations rédactionnelles}

Il est notoire, dans le cinéma français des années quarante et cinquante, que toute scénarisation commence par une conversation. C'est un topos admirablement développé dans le film scénarisé par Henri Jeanson, La Fête à Henriette (Julien Duvivier, 1952), mettant en scène deux scénaristes à l'œuvre. Jeanson considérait comme un trait distinctif français, le travail du scénario « à la bonne franquette », traditionnellement abattu dans des hôtels campagnards loin de la capitale ${ }^{44}$. Pierre Bost

41. Comme le souligne Claire Bustarret : «Collages, découpages et bricolages témoignent de la coexistence, au cœur des processus de genèse, d'une pensée du virtuel et d'une manipulation du matériel »; Claire Bustarret, « Découpage, collage et bricolage : la dynamique matérielle du brouillon moderne », dans L'Imaginaire de l'écran/Screen Imaginary, dir. Nathalie Roelens et Yves Jeanneret, New York, Rodopi, 2004, p. 120. 42. La correspondance joue un rôle tout aussi important dans les négociations rédactionnelles.

43. Continuité dialoguée dactylographiée, p. 1-2, p. 6 (95/1 A4.1).

44. Henri Jeanson, dans Jeux d' auteurs..., op. cit., p. 259. Dans le registre de la dispute, Jean Renoir évoquait, quant à lui, une « espèce de discussion [de laquelle] naît la matière vivante du film », voir à ce propos Olivier Curchod, « La "Méthode Renoir" et ses légendes : petite histoire d'un casting "provocateur" », Genesis, n² 28, « Cinéma », 2007, p. 73-87. 
en résume la trame dans une conférence sur le métier de scénariste :

Cela fait, il se produit un phénomène invariable aussi régulier, aussi inéluctable que les migrations des oiseaux, et qui du reste est du même ordre : les auteurs du film partent pour la campagne. [...] Oui, il vaut mieux quitter Paris. Pourquoi ? Parce que le travail de cinéma est un travail d'équipe, et qu'il est pratiquement impossible de réunir régulièrement et longtemps, dans Paris, deux ou trois personnes, dont chacune a ses occupations. [...] Nous voici à la campagne. Et maintenant commence le travail du scénario. C'est le même stade où un romancier qui n'a encore qu'une idée, un sujet, se met devant sa table et écrit. La différence est que, pour un film, l'auteur - au départ - est toujours deux, et quelquefois trois et que les premiers temps du travail se passent en conversations. C'est un des mystères de ce métier et l'une de ses nécessités. L'homme de cinéma a peur de la solitude ${ }^{45}$.

Dans le cas de Douce, une partie de ces réunions se seraient déroulées à l'hôtel du Plat-d'étain à Houdan, si l'on en croit l'en-tête d'un papier à lettres ${ }^{46}$. En plus d'être conviviales, les discussions stimulent la création et déjouent les impasses, comme celle à laquelle était confronté Aurenche pour les dialogues au début de l'année 1943, peu avant l'arrivée de Bost ${ }^{47}$. Avec « des délais [de création] toujours trop courts 48 », l'échange oral est un véritable facteur de productivité. Aurenche le considère comme une technique infaillible, au risque de manquer à la paternité des idées, puisque les conversations n'ont pas d'auteurs :

C'est comme ça qu'arrivent les bonnes idées : quelqu'un lance un mot, l'autre le fait rebondir. Allez savoir, après, qui des deux ou des trois est le véritable auteur !

Aurenche ne croit d'ailleurs pas si bien dire (ou plutôt écrire). Dans son entretien avec Alain Riou, il prête l'origine d'une réplique à Bost :

J'ai fait appel à mes souvenirs, mais la contribution de Bost est capitale. Il a ajouté des répliques formidables comme l'une des premières que prononce Marguerite Moreno : «Il fait froid comme en temps de guerre ${ }^{49}$. »

Et pourtant, d'après les archives scénaristiques, la réplique est bel et bien de la main d'Aurenche ${ }^{50}$. Sans retranscriptions des interactions orales qui ont eu cours durant la ge- nèse, il est impossible de trancher en faveur d'une attribution ou d'une autre, sans se demander dans quelle mesure Aurenche aurait pu coucher sur le papier ce que Bost, ou même une fulgurance collective, a créé51. En conséquence, il paraît prudent de prendre en compte l'éventuelle complémentarité des pratiques orales et écrites dans le contexte d'une genèse scénaristique à plusieurs ${ }^{52}$. L'hypothèse suivante peut être émise au préalable : l'oral combine des qualités de spontanéité, d'éclaircissement et de civilité, il favorise les négociations ; l'écrit, quant à lui, administre et entérine des processus, offre un support de référence et de stockage, une archive consultable à distance dans le temps et dans l'espace53. Il n'est pas non plus à exclure que les modalités de la conversation orale se combinent à celles de la genèse écrite de différentes manières : à travers

45. Nous soulignons. Pierre Bost, brouillon non daté intitulé « Conférence Cinéma A.O.F », p. 9-10, fonds privé. Nous remercions Jérôme Bost de nous avoir ouvert l'accès à ses archives familiales.

46. 95/2, A4.1

47. Comme le relève Denis Apothéloz, lors d'une conversation rédactionnelle, la progression du texte repose sur une dynamique interactionnelle, où « toute marque d'incomplétude est en réalité une invitation à projeter » ; Denis Apothéloz, «Progression du texte dans les rédactions conversationnelles : les techniques de reformulation dans la fabrication collaborative du texte », dans Robert Bouchard et Lorenza Mondada (dir.), Les Processus de la rédaction collaborative, Paris, L'Harmattan, 2005, p. 180.

48. P. Bost, « Conférence Cinéma A.O.F », p. 12.

49. La Suite à l'écran, op. cit., p. 224, p. 91.

50. 94/9 A4.1.

51. Bien souvent, le passage à l'inscription gomme toute prise en charge individuelle d'un corédacteur dans une forme de stabilisation impersonnelle et greffière du texte ; Marie-Madeleine de Gaulmyn, « Processus de reformulation dans des tâches d'écriture collective », dans Anne-Claude Berthoud et Lorenza Mondada (dir.), Modèles du discours en confrontation, Bern, Peter Lang, 2000, p. 79-96.

52. Bien que l'articulation entre oralité et scripturalité, ainsi que les genèses en collaboration, soient autant de cas limites pour les études génétiques, comme l'a énoncé Almuth Grésillon, «"Nous avançons toujours sur des sables mouvants." Espaces et frontières de la critique génétique », dans La Création en acte. Devenir de la critique génétique, dir. Paul Gifford et Marion Schmid, Amsterdam/New York, Éditions Rodopi, 2007, p. 33-35.

53. Outre les travaux pionniers de Marie-Madeleine de Gaulmyn sur la rédaction conversationnelle, la linguistique et la sociologie du travail investissent ce champ de recherches fécond. Voir Michèle Lacoste et Michèle Grosjean, «L'oral et l'écrit dans les communications de travail, ou les illusions du "tout-écrit"», Sociologie du travail, n 4, 1998, p. 439-464. 

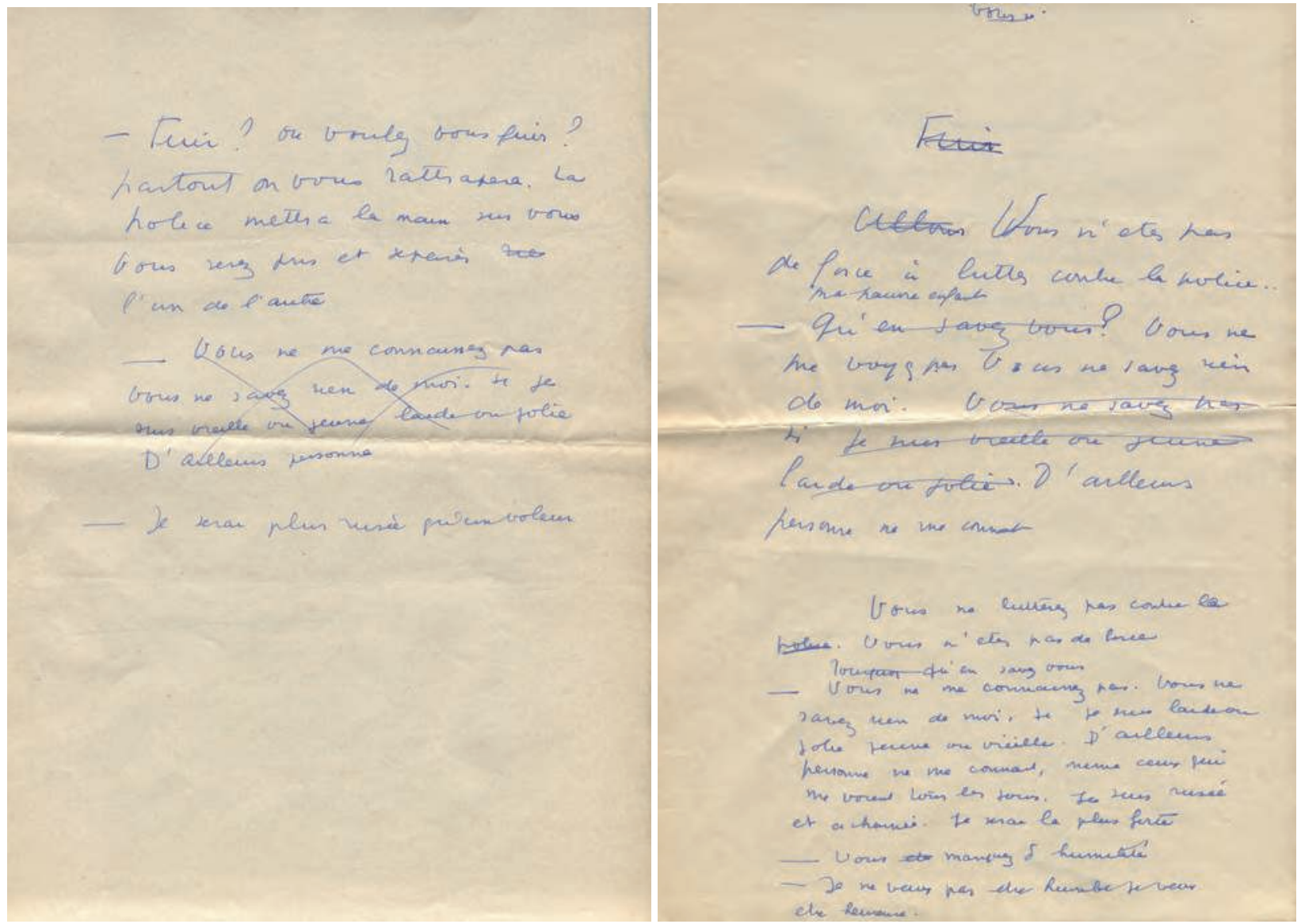

Fig. 6 (a-b) : Extrait des brouillons d'une continuité dialoguée de la main de Jean Aurenche, 1942. Conservé dans le fonds Claude Autant-Lara (CSL.5), 94/9 A4.1, Collection Cinémathèque suisse

l'élaboration soignée des dialogues (sans doute dits, voire joués par les auteurs ${ }^{54}$ ), l'enchaînement de tours d'écriture avec l'exigence d'un achèvement par la lecture et le commentaire à voix haute, un souci de complétude et de clarté qui préfère le repentir oral à la rature scripturale. Tous ces cas de figure dépendent d'un principe de coopération qui nécessite des accommodations entre les auteurs, sans commune mesure avec le fantasme d'une intransigeance auctoriale 55. En ce sens, Aurenche, Bost et Autant-Lara, par leur capacité d'adaptation les uns aux autres, deviendront les «professionnels intégrés 56 » parmi les plus productifs de l'industrie cinématographique française dans la décennie suivante.
54. Ce sera le cas, dix ans plus tard, pour la genèse de La Traversée de Paris. On le sait grâce à des éléments de dialogues sténographiés par un tiers dans une continuité dialoguée (196/4 A4.1).

55. Concernant la genèse de Douce et d'après les sources, nous nous inscrivons en faux contre l'argument de François Albera sur une « volonté de contrôle total » de la part d'Autant-Lara, voir « Claude Autant-Lara, portrait en auteur contrarié », dans L'Auteur de cinéma : histoire, généalogie, archéologie, Paris, Association française de recherche sur l'histoire du cinéma, 2013, p. 100. Il paraît peu prudent de confondre l'obsession de la planification, la volonté de construire une œuvre et la constitution d'archives. La première gagne Autant-Lara après ses déboires avec le producteur Paul Graetz pour Le Diable au corps (voir 92/1 A7, 92/2 A7, CSL.5 ; La Suite à l' écran, op. cit., p. 142-143) ; la deuxième correspond surtout à des propos réactionnaires qu'il adopte à travers ses essais dès les années soixante, s'inventant une posture d'auteur et biaisant avec de nombreux faits ; la troisième, malgré une conservation procédurière, où il faut reconnaître un symptôme quasi paranoïaque, révèle aussi un nombre considérable de documents qui font la part belle au travail de ses collaborateurs.

56. Dans la mesure où ils font appel à leurs « aptitudes sociales » et à leur « connaissance des conventions »; H. Becker, op. cit., p. 238-242. 
Adrien Gaillard (1986) est assistant-doctorant en linguistique à la section de français moderne de l'université de Lausanne. Il effectue des recherches dans le cadre du projet du Fonds national suisse qui s'intitule «Discours du scénario : étude historique et génétique des adaptations cinématographiques de Stendhal ». Il rédige une thèse sur les enjeux génétiques, textuels, énonciatifs et stylistiques du scénario, intitulée provisoirement «Le scénario de film, entre écrire et projeter : étude linguistique des adaptations de la "Qualité française" ».

adrien.gaillard@unil.ch

Julien Meyer (1985) est assistant-doctorant à la section d'histoire et esthétique du cinéma de l'université de Lausanne. Il effectue des recherches dans le cadre d'un projet financé par le Fonds national suisse susmentionné. En parallèle, il rédige une thèse dans une approche historique et sociologique sur le métier de scénariste dans le cinéma français de l'immédiat après-guerre.

julien.meyer@unil.ch

Résumés

\section{Jean Aurenche, Pierre Bost et Claude Autant-Lara, auteurs de Douce. Genèse d'une pratique scénaristique}

À la lumière des archives du fonds Claude Autant-Lara déposé à la Cinémathèque suisse, cet article aborde la naissance d'une collaboration entre le réalisateur et le tandem de scénaristes Jean Aurenche et Pierre Bost, à travers la genèse scénaristique du film Douce (19421943). Les discours de réception désignent habituellement «Aurenchébost » comme s'il s'agissait d'un auteur unique avec une méthode de création. Il convient de reconsidérer leur travail dans l'historicité et la contingence des pratiques d'écriture. À ce titre, les documents scénaristiques procèdent à la fois d'une dimension processuelle - un texte en devenir - et d'une dimension interlocutive - le fruit d'une activité concertée. Cette dernière implique, entre autres, un jeu de formatage et de pagination dans la rédaction de la continuité dialoguée et du découpage technique. Enfin, la scénarisation ne se limite pas à des tours d'écriture mais doit être également considérée en termes de rédactions conversationnelles.

Based on Claude Autant-Lara's archives deposited at the Swiss film library, this article studies the birth of a collaboration between the director and the duo of screenwriters, Jean Arenche and Pierre Bost, through the screenwriting genesis of the film Douce (1942-1943). Usually, reviews refer to "Aurenchébost" as if they were a single author with a creative method. Their work should be reconsidered within the historicity and contingency of writing practices. As such, the scriptwriting documents are the result of a processing dimension $-\mathrm{a}$ text in the making - and a dialogical dimension - the outcome of a concerted activity. The latter implies, among other things, a play with the formatting and pagination in the scenario's composition and technical breakdown. Finally, their screenwriting is not limited to writing each in turn but must also be considered in terms of conversational writing.

Im Lichte der Archivmaterials des Nachlasses von Claude AutantLara, das sich in der Schweizer Cinémathèque befindet, geht der vorliegende Artikel der Entstehung einer Kooperation nach, nämlich jener zwischen dem Regisseur Claude Autant-Lara und den beiden Drehbuchautoren Jean Aurenche et Pierre Bost im Rahmen der Genese des Films Douce (1942-1943). Die bisherige Rezeption insinuierte, dass es sich bei „Aurenchébost“ um einen einzigen Autor mit nur einer Methode gehandelt hätte. Man muss aber die Arbeit von Aurenche und Bost in ihrer Historizität und in der Kontingenz der Schreibpraxis neu betrachten. Dabei zeigt sich, dass die Drehbuch-Dokumente zugleich eine prozessuale Dimension haben - wir finden einen Text in seinem Werden vor - und dass sie auch eine dialogische Komponente aufweisen - sie sind die Frucht einer gemeinsamen Tätigkeit. Unter anderem bedeutet dies ein Spiel der Formatierung und der Paginierung im Rahmen der Redaktion der Dialoge und des technischen Drehbuchs. Schließlich beschränkt sich die Ausarbeitung des Szenarios nicht auf die Erstellung abwechselnder Schreiber, sondern muss ebenso hinsichtlich der Redaktion der Dialoge betrachtet werden.
Basandosi sugli archivi del fondo Claude Autant-Lara conservati alla Cineteca svizzera, quest'articolo affronta la nascita di una collaborazione tra il regista e il tandem di sceneggiatori Jean Aurenche e Pierre Bost, attraverso la genesi della sceneggiatura del film Douce (19421943). La critica e il pubblico sono soliti riferirsi a "Aurenchébost" come se si trattasse di un unico autore con un unico metodo di creazione. Se si riconsidera il loro lavoro nella storicità e nella contingenza delle pratiche di scrittura, ci si accorge tuttavia che i documenti delle sceneggiature derivano allo stesso tempo da una dimensione processuale - un testo in divenire - e da una dimensione interlocutiva - il frutto di un'attività concertata. Quest'ultima implica, tra le altre cose, un meccanismo di formattazione e d'impaginazione nella redazione della continuità dei dialoghi e delle suddivisioni tecniche. Infine, la creazione della sceneggiatura non si limita soltanto a sessioni di scrittura ma deve essere considerata anche in termini di redazioni conversazionali.

A partir do espólio de Claude Autant-Lara depositado na Cinemateca Suiça, este artigo examina a colaboração entre o diretor e o tandem de argumentistas Jean Aurenche e Pierre Bost, que levou à criação do argumento do filme Douce (1942-1943). Refere-se geralmente "Aurenchébost" como se fosse um autor único com um método de criação. Deve reavaliar-se o seu trabalho na historicidade e na contingência das práticas de escrita. Como tal, os documentos do argumento possuem ao mesmo tempo uma dimensão processual um texto em construção - e uma dimensão de interlocução - fruto de uma atividade concertada. Esta última implica, nomeadamente, um exercício de formatação e paginação na redação da continuidade do diálogo e na montagem técnica. Assim, a cenarização não se limita a ser uma alternãncia de atos de escrita, mas deve mesmo ser considerada como uma redação em termos dialogais.

A la luz de los archivos del fondo Claude Autant-Lara depositados en la Cinemateca suiza, este artículo aborda el nacimiento de una colaboración entre el director y el tándem de guionistas Jean Aurenche y Pierre Bost, a través de la génesis del guión de la película Douce (1942-1943). Los discursos de recepción designan al dúo de guionista "Aurenchebost", como si se tratara de un único autor con un solo método de creación. Es necesario reconsiderar su trabajo en la historicidad y la contingencia de las prácticas de escritura. En esta perspectiva, los documentos guionísticos proceden a la vez de una dimensión procesal -un texto en construcción- y de una dimensión interlocutiva -el fruto de una actividad concertada. Esta última implica, entre otros, un juego de formateo y de paginación en la redacción de la continuidad dialogada y de la segmentación técnica. Finalmente, la elaboración de un guión no se limita a una escritura alternada sino que debe se considerada también en términos de redacciones conversacionales. 

Engagements créatifs Luciano Berio, Heinz Holliger et la genèse de la Sequenza VII pour hautbois

Nicolas Donin

$\mathrm{L}^{\prime}$

9 interaction entre compositeur et interprète a connu de multiples figures historiques, de la relation fusionnelle entre Olivier Messiaen et la pianiste Yvonne Loriod (successivement son élève en composition, sa partenaire au concert, sa muse, son épouse, son imprésario officieux et l'éditrice officielle de ses écrits posthumes) à la trahison créatrice de Jacques Hétu par Glenn Gould ${ }^{1}$, en passant par les duos artistiques et amicaux que l'on a pris l'habitude d'associer à des œuvres marquantes du répertoire : Niccolò Paganini et Hector Berlioz (Harold en Italie), Joseph Joachim et Johannes Brahms (Concerto pour violon op. 77), Georges Barrère et Edgard Varèse (Density 21.5), Robert Craft et Igor Stravinsky (œuvres de sa période sérielle), etc.

Dans la musique d'avant-garde de l'après-Deuxième guerre mondiale, dont l'économie symbolique (puis l'historiographie) a reposé essentiellement sur des « auteurs » démiurges de l'innovation radicale, la spécialisation et la virtuosité de l'interprète ont été exacerbées. La difficulté des œuvres nouvelles composées par la génération née dans les années vingt - celle de Pierre Boulez, Luigi Nono ou Karlheinz Stockhausen - n'a pu être surmontée et acceptée que grâce à un certain nombre d'interprètes hors du commun prêts à développer toute une nouvelle technique de lecture et de jeu pour ce répertoire. Certains noms sont souvent cités du fait de leur engagement intensif au service des œuvres nouvelles de cette époque : le pianiste David Tudor, la mezzo-soprano Cathy Berberian, le percussionniste Christoph Caskel, pour ne citer que quelques-uns des héros cosmopolites associés au développement foudroyant des langages et des esthétiques entre les années cinquante et soixante-dix.

Si le rôle de ces instrumentistes dans la vie musicale européenne et nord-américaine a été éminent, il n'en est pas de même de leur place dans la littérature spécialisée, qui a rarement étudié leur contribution à la création autrement qu'au prisme monographique des vies de compositeurs, dans lesquelles ils apparaissent le plus souvent comme de fidèles serviteurs - voire de simples figurants. Ce serait en quelque sorte leur engagement par tel ou tel qui justifierait de les étudier, plutôt que leur engagement (personnel, existentiel) dans la création musicale ${ }^{2}$. Pourtant, une étude sans a priori de la gestation des œuvres auxquelles leur nom est associé montrerait qu'ils ont souvent apporté bien davantage à la conception qu'une performance sportive finale, comme nous allons nous efforcer de le montrer à partir de l'exemple d'une des célèbres Sequenze de Luciano Berio (1925-2003) - série d'œuvres solistes pour différents instruments composée entre 1958 et $2002^{3}$. Si nous ne pouvons, dans l'espace de cet article, proposer une reconstruction détaillée du processus de création de la Sequenza VII (1969) pour hautbois, nous en retiendrons plusieurs aspects saillants qui permettent d'esquisser une figure de la collaboration compositeur/interprète potentiellement valable pour d'autres Sequenze et pour d'autres œuvres de musique dite contemporaine.

1. Dans un de ses disques, le pianiste canadien interpréta une partition de son jeune compatriote, sans l'avoir jamais rencontré ni même prévenu, en s'écartant librement de la lettre du texte. Voir le témoignage post-traumatique de Jacques Hétu : «Variations et variantes », dans Ghyslaine Guertin (dir.), Glenn Gould pluriel, Verdun (QC), Louise Courteau, 1988. 2. Parmi les rares exceptions à cette logique, notons par exemple le chapitre consacré à la violoncelliste Charlotte Moorman par Benjamin Piekut dans Experimentalism Otherwise. The New York Avant-Garde and its Limits (Berkeley, University of California Press, 2011).

3. Sur les Sequenze, voir Philippe Albèra, « Introduction aux neuf sequenzas », Contrechamps, ${ }^{\circ}$ 1, 1993, p. 91-122 et Janet K. Halfyard (dir.), Berio's Sequenzas: Essays on Performance, Composition and Analysis (Aldershot/Burlington, Ashgate, 2007). 


\section{L'interprète vu par le compositeur}

En 1970 paraît un 33 tours enregistré en 1969 intitulé The spectacular Heinz Holliger plays music by Berio Castiglioni Holliger Huber Krenek Lehmann (fig. 1), composé d'œuvres nouvelles pour hautbois solo ou accompagné suscitées par Heinz Holliger (né en 1939), lauréat du concours international de Genève en 1959, infatigable promoteur de son instrument à travers la redécouverte de répertoires anciens et le développement d'un nouveau répertoire tirant parti de la grande variété de ses modes de jeu, souvent mal connue des compositeurs. Des dizaines de partitions nouvelles dont Holliger s'est fait l'ambassadeur, la Sequenza VII est généralement considérée comme l'un des fleurons, et sa place est désormais éminente au sein de la littérature pour hautbois.

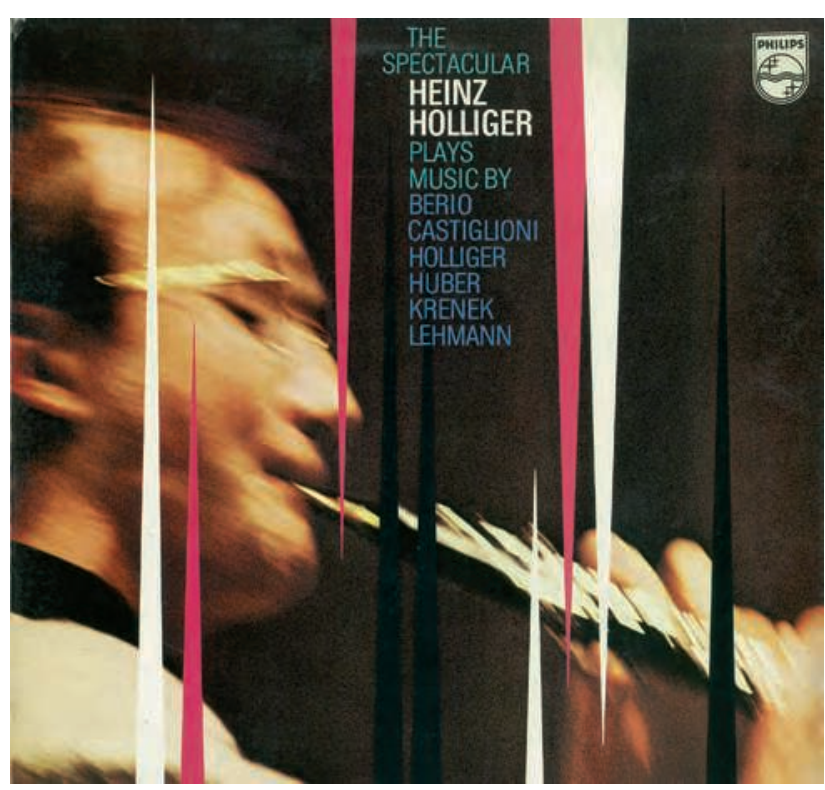

Fig. 1 : Pochette du disque The spectacular Heinz Holliger plays music by Berio Castiglioni Holliger Huber Krenek Lehmann (Philips, 1970)

À rebours de l'argument de vente de l'éditeur phonographique, ce n'est pas la virtuosité « spectaculaire » de Holliger que Luciano Berio met en avant dans sa présentation de Sequenza VII reproduite au dos de la pochette :

Between the virtuosi and the best soloists of our time, there are striking and radical differences, which mirror the profound changes in the music of the last 60 years. Today the modern soloist - like every modern researcher in every field - both needs and is able to have an extremely broad angle of vision over historical time. He can interpret the experiences of the past as well as those of the immediate present. In contrast to the virtuoso, he can master extensive historical perspectives, since he uses his instrument not only as a means of pleasure, but of insight (of intellectual analysis). So he is in a position to collaborate in the music and contribute to it, instead of "serving" it with false humility. By this I simply mean to say that my piece "Sequenza VII" was written with this kind of interpreter - Heinz Holliger - in mind . $^{4}$

Le propos est caractéristique du discours de Berio sur ses Sequenze ; ce même fragment textuel est d'ailleurs repris au fil des ans (avec des variations) pour accompagner d'autres Sequenze et rendre hommage à leurs interprètes respectifs 5 . Il y est question d'un dépassement de la « virtuosité » (critiquée pour son hédonisme rivé à l'instant présent), d'une intellectualité de l'instrumentiste (qualifié de « chercheur »), d'une « collaboration » de l'interprète à la musique, d'un Holliger présent à l'esprit de Berio au cours de la composition qui lui est dédiée. Tous ces marqueurs de l'engagement créatif, s'ils sont assurément cohérents avec la physionomie artistique de Holliger, ne disent néanmoins pas tout de sa contribution à la Sequenza VII car ils semblent ici ne qualifier que la phase de l'exécution de l'œuvre - la réception et l'appropriation du texte musical par l'interprète puis sa performance publique. Se tourner vers le processus de création permettra tout à la fois de nuancer la parole de Berio (donc également le discours musicologique, qui s'en est largement inspiré) et de montrer en quoi, dans le même temps, elle reflète au fond l'accord entre compositeur et interprète sur leurs rôles respectifs.

Il est bien connu, au sujet de Sequenza VII, que Holliger ne s'est pas cantonné à figurer pour l'« esprit » du compo-

4. Citation de Berio au sein des «Notes compiled by Heinz Holliger », pochette du disque Philips 6500 202, verso. Je remercie Sonia Popoff de m'avoir facilité l'accès à l'exemplaire conservé à la Médiathèque musicale Mahler (Paris).

5. Voir également les formulations plus amples, plaidant pour une « virtuosité de la conscience », données dans : Luciano Berio, Entretiens avec Rossana Dalmonte. Écrits choisis, éd. Martin Kaltenecker, Genève, Contrechamps, 2010, p. 70-76. 
siteur un type idéal d'interprète auquel son œuvre mériterait d'être confiée. La considération des premières mesures de la partition suffit à se convaincre que l'inscription de la personne du dédicataire dans l'œuvre est plus profonde. Sequenza VII est en effet structurée autour d'une note, le si médian de clé de sol (désormais désigné ici si ${ }^{3}$ suivant les conventions francophones), qui, comme l'a rappelé Paul Roberts, croise deux types de caractéristiques : d'une part, c'est la note que le hautbois peut produire par le plus grand nombre de doigtés différents, autorisant le riche jeu de variation de timbres auquel est consacrée toute la première minute de l'œuvre ; d'autre part, cette note est désignée par la lettre $\mathrm{H}$ dans le solfège germanophone, autrement dit les deux premiers « $\mathrm{H} »$ claironnés triple forte par l'instrument peuvent être compris comme une inscription de la dédicace à $\mathrm{H}$ [einz] $\mathrm{H}$ [olliger] à même le texte musical6 ${ }^{6}$ Rien ne prédisposait Berio, peu familier du hautbois, à connaître la multiplicité de variations de timbres et de doigtés permise par ce $s i^{3}$. Ce sont ses échanges préalables avec l'interprète qui lui ont permis de la cibler et de l'exploiter. Car la « collaboration » de l'interprète à la musique, évoquée plus haut par Berio, a revêtu un caractère très concret dès le projet de l'œuvre. Il est possible de la retracer sur la base d'un dossier génétique centré sur les courriers très détaillés adressés par Holliger à Berio, et intégrant un certain nombre d'autres avant-textes de la Sequenza VII - tous documents conservés à la Fondation Paul Sacher (Bâle)7. S'y ajoutent des précisions et des compléments apportés par Heinz Holliger, au regard d'un sous-ensemble du dossier génétique, lors de l'entretien qu'il nous a accordé à son domicile bâlois le 4 décembre 2014.

Passons donc de l'aval à l'amont de l'œuvre - c'està-dire aussi, dans un premier temps, du point de vue du compositeur à celui de l'interprète.

\section{Le compositeur vu par l'interprète}

Le point de départ du projet de Sequenza VII n'est ni une initiative de Berio, ni le souhait d'un programmateur de concerts, mais une requête de la part de Holliger. La lettre qui ouvre la correspondance entre les deux musiciens indique bien comment Holliger pré-voit en Berio l'auteur possible d'une Sequenza pour hautbois :

\section{Cher Monsieur Berio,}

depuis longtemps déjà j'admire votre musique. C'est pourquoi j'ose vous demander si vous seriez intéressé à écrire une courte pièce pour moi, soit pour hautbois seul, soit pour hautbois et harpe (ma femme est harpiste et a déjà joué votre Sequenza II) soit pour hautbois, harpe et un 3ème instrument (percussion, cimbalum, clavecin, flûte p.e.). Je pense à une pièce dans le caractère des SEQUENZE. Comme vous savez peut-être j'ai développé beaucoup de possibilités nouvelles sur mon instrument et j'ai déjà reçu beaucoup de pièces qui se servent de cette nouvelle technique. (p.e. Penderecki, Stockhausen, Henze, Krenek, Castiglioni, Kagel etc.). Je ne suis pas très riche et je ne pense vous offrir pour cette pièce (d'une durée de 4' à 7') que 2000 frs suisses. $[\mathrm{M}]$ ais je vous rassure que je jouerai beaucoup votre œuvre et que je l'enregistrerai sur disque si possible. Écrivez-moi si cette proposition vous intéresse. Je vous enverrai alors une liste avec toutes les possibilités du hautbois. - Pourriez-vous écrire cette pièce déjà en 1967 ? Je pourrais la mettre alors sur mon disque que je fais avec Philips $[\ldots]^{8}$.

6. Paul Roberts, «On Luciano Berio's Sequenza VII for oboe », Mitteilungen der Paul Sacher Stiftung, n 16, 2003, p. 36-41. En dehors de cette brève contribution, les deux principaux travaux sur la Sequenza VII basés sur les sources sont : Thomas Gartmann, «... dass nichts an sich jemals vollendet ist. "Untersuchungen zum Instrumentalschaffen von Luciano Berio, Berne, Haupt, 1997 ; et Anne C. Shreffler, « Circuits de collaboration. Heinz et Ursula Holliger », dans Ulrich Mosch et Matthias Kassel (dir.), «Entre Denges et Denezy... » : la musique du XX e siècle en Suisse, manuscrits et documents, Bâle, Paul Sacher Stiftung, 2001, p. 106-116.

7. Collections Berio, Globokar et Holliger. Nous remercions respectivement Angela Ida De Benedictis, Robert Piencikowski et Heidi Zimmermann, ainsi que Michèle Noirjean, pour leur accueil et leur disponibilité à Bâle. La langue des échanges entre les trois musiciens est le français, qu'ils parlent couramment. Nous conservons dans la mesure $\mathrm{du}$ possible les particularités typographiques et grammaticales des documents cités, en suivant les conventions éditoriales usuelles. Concernant les lettres de Berio à Holliger, actuellement non localisables, nous nous basons sur les informations et transcriptions rassemblées par Anne C. Shreffler lors de son étude précédemment référencée. Enfin, nous remercions Talia Pecker Berio et Heinz Holliger d'avoir donné leur autorisation pour la citation et la reproduction des documents discutés dans la présente étude.

8. Lettre de Holliger à Berio datée du 29 mars 1967. Fondation Paul Sacher, Collection Luciano Berio, correspondance. Une reproduction figure dans Anne C. Shreffler, art. cité, p. 108. 
Lorsque Holliger écrit à Berio, cinq Sequenze sillonnent déjà la vie musicale. La cinquième pour trombone, dernière en date, est fréquemment jouée en Europe par Vinko Globokar. La sixième pour alto (composée précisément en 1967) est destinée à Serge Collot. Ces deux musiciens sont de proches collègues et amis de Holliger. Tous trois sont virtuoses d'instruments peu dotés en répertoire et en lesquels ils voient un potentiel d'inventivité organologique et sonore largement sous-exploité. Globokar et Holliger sont également compositeurs (le premier a reçu l'enseignement de Berio, le second de Boulez, parmi d'autres figures marquantes) ; leurs premières compositions, particulièrement pointues dans l'exploration des instruments à vent, sont en partie liées à la recherche instrumentale qu'ils mènent en même temps. La Sequenza VII est donc autant le projet de Holliger en tant qu'interprète engagé que de Berio en tant que compositeur.

Mais, pourrait-on objecter, la proposition et sa réalisation adviennent elles-mêmes sur le fond d'un projet compositionnel qui les rend possibles : l'idée d'une série de solos intitulés Sequenza, préférablement monodiques, explorant un ou plusieurs idiomatismes instrumentaux avec l'aide d'un chercheur-virtuose. Ce projet a défini un champ d'intervention dans lequel Holliger trouve spontanément sa place et est prêt à jouer son rôle, pluriel, de conseiller-interprète-ambassadeur - et pour commencer, de commanditaire. Néanmoins, là encore, le projet des Sequenze est à la fois celui de Berio - cela va de soi - mais aussi, dès l'origine, celui de ses interprètes. En effet, la Sequenza pour flûte (1958), dont le succès a certainement incité le compositeur à prolonger l'expérience isolée au sein d'une série numérotée, était elle-même la réponse à une sollicitation de Severino Gazzelloni qui réclamait à son ami, au début de l'été 1958 , « une petite pièce pour "flûte solo" d'une durée d'environ 4 ou 5 minutes à inclure dans le programme : Togni - Messiaen - Berio - Boulez ${ }^{9} »$, pour un récital qu'il devait donner à Cologne en septembre. Cette fonction motrice de l'interprète ne fera que s'accentuer au fil du temps, jusqu'au point où Berio se trouvera en quelque sorte harcelé de propositions instrumentales d'interprètes prêts à tout pour avoir «leur» Sequenza.

\section{Proposer, disposer}

L'accord de principe de Berio, par retour de courrier, amorce une série d'échanges qui vont accompagner la genèse du projet puis de la partition, elle-même composée rapidement et dont aucune esquisse directe ne nous est connue.

Entre avril 1967 et avril 1968, Holliger fait parvenir à Berio deux documents représentant l'état le plus avancé de description des possibilités de son instrument. D'une part, une copie de la liste qu'il a lui-même établie au début des années soixante, intitulée «Tabelle neuer Möglichkeiten der Oboe » (table des nouvelles possibilités du hautbois), où sont indiquées les principales caractéristiques du hautbois en termes de tessiture, dynamique, trilles, sons harmoniques, etc. ${ }^{10}$. D'autre part, le premier traité des «nouveaux sons » des instruments à vent alors fraîchement paru, dont l'auteur, le violoniste et compositeur florentin Bruno Bartolozzi, avait divisé l'étude en "possibilités monophoniques » et «possibilités multiphoniques 11 », renouvelant avec force l'enjeu polyphonique dans l'écriture de ces instruments monodiques - y compris par l'exemple, puisqu'il joint à son traité, sous forme de partition et de disque 45 tours, des fragments démonstratifs pour chaque instrument composés par ses soins.

De Bartolozzi, à supposer qu'il l'ait lu de près, Berio ne retient pas la focalisation sur les sons multiphoniques

9. Lettre de Gazzelloni à Berio datée du 7 juillet 1958. Fondation Paul Sacher, Collection Luciano Berio, correspondance. Nous remercions chaleureusement Angela Ida De Benedictis de nous avoir communiqué sa transcription de plusieurs passages de cette correspondance - et, plus généralement, d'avoir relu le présent article avec autant d'exigence que de générosité.

10. Holliger date cette liste des premières années où il passe commande d'œuvres nouvelles à des compositeurs, probablement en 1961 (la première de cette longue série étant la Sonate pour hautbois et piano de Milko Kelemen, 1960). Dans une lettre à Berio du 21 juin 1967, Holliger indique avoir découvert «maintes choses intéressantes qui ne sont pas encore sur la liste ». Il transmettra en effet à Berio, à un stade ultérieur, une « Liste supplémentaire pour le hautbois » incluant notamment une série de doigtés produisant des sons multiphoniques.

11. Bruno Bartolozzi, New sounds for woodwind (trad. angl. Reginald Smith Brindle), London, Oxford University Press, 1967. 
(qu'il utilisera très parcimonieusement dans Sequenza VII) mais un fait organologique plus général et beaucoup moins contraignant sur le plan harmonique : l'abondance de doigtés résultant de « toutes les combinaisons possibles des doigts et des trous multipliés par toutes les combinaisons des clés chromatiques 12 ». En effet, la principale question adressée par Berio à Holliger en rapport avec le projet d'œuvre porte sur la note du hautbois qui pourrait être émise par le plus grand nombre de doigtés différents. Berio n'explicite pas le motif de cette requête, mais Holliger n'aura pas eu de mal à le rattacher à une préoccupation pour le timbre, qui guide également l'enquête de Bartolozzi. Ce dernier précisait son « objectif premier » dans les termes suivants, au seuil du chapitre «monophonique» de son traité : assurer «l'émission de sons dotés d'une différenciation de timbre maximale 13 ». Dans la lettre du 29 avril 1968 où il lui donne pour principale réponse le $s i^{3}$ (qui sera effectivement retenu), Holliger renvoie à son tour Berio au livre de Bartolozzi : « [il] est excellent sur ce point, vous y trouverez beaucoup de matériel14. »

Mais Berio n'est pas en train de s'engager sur la voie d'un long apprentissage solitaire des particularismes complexes du hautbois - un instrument qui ne l'a guère inspiré jusqu'alors, comme Holliger se souvient qu'il le lui avait confié sans faux détour. De l'abondance de doigtés de $s i^{3}$ disponibles, il tire l'idée qu'il est possible de réaliser une riche mélodie de timbres sur une seule note15 et adoptera, dans son travail compositionnel, une distinction entre cinq timbres distincts, notés par les chiffres cerclés de 1 à 5 placés au-dessus du si dans la partition, dont les modalités de réalisation (c'est-à-dire le choix des doigtés) seront tacitement laissées à l'appréciation de l'interprète.

Qu'a sélectionné Berio dans la «Tabelle neuer Möglichkeiten der Oboe » proposée par Holliger ? Nous disposons, pour répondre, d'un élément précieux à la datation incertaine : les annotations portées par Berio sur son propre exemplaire de la liste, conservé à la fondation Sacher (fig. 2). La correspondance laisse à penser que l'unique séance d'expérimentation commune entre les musiciens à Milan a pu avoir lieu fin avril ou début mai 1969, période d'écriture de la pièce. En plus d'enregistrer Holliger sur bande ${ }^{16}$, Berio aurait gardé trace des éléments les plus intéressants de la liste en les entourant. Le recto a été reproduit pour la première fois par Anne Shreffler, qui relevait l'annotation «trillo molto buono » à propos d'un trille de double harmonique ${ }^{17}$. La brève annotation portée par Berio sur la deuxième page n'est sans doute pas moins significative pour la genèse de l'œuvre : le compositeur y pointe la ligne consacrée aux effets de sforzando (sfz-Effekte). Holliger y donne plusieurs exemples de succession rapide d'un son fortissimo et d'un son harmonique pianissimo. Ce contraste d'attaque, de dynamique et de timbre produit un effet de rebond, voire d'écho, qui s'avérera primordial dans la Sequenza. La cellule iambique sur $s i^{3}$ qui constitue le premier exemple de sforzando noté dans la liste se retrouvera, presque littéralement, au commencement de l'œuvre donnée en première audition par Holliger à la Biennale de Zagreb le 15 mai suivant.

Si Berio n'a pas consenti à la plongée expérimentale dans l'instrument à laquelle Holliger l'incitait (et que ce dernier pratiquait parallèlement avec leur ami commun Vinko Globokar, en ces premiers mois de 1969, pour l'élaboration de Discours III qui lui est également dédié), il n'en reste pas moins que le compositeur a travaillé en dialogue avec le jeu et les savoirs du hautboïste dès l'abord du projet d'œuvre et jusqu'au moment de sa rédaction. Comme nous allons le voir, ce dialogue n'a pas non plus pris fin avec la mise au net de la partition.

12. Ibid., p. 7.

13. Ibid., p. 12 (emphase de l'auteur).

14. En précisant dans la même lettre que le livre contient « déjà une trentaine de doigtés », Holliger se réfère implicitement à deux passages : l'un est une liste de huit doigtés sur $s i^{3}$ classés par qualité de timbre (p. 20) ; l'autre est un extrait de la partie de hautbois d'une composition de Bartolozzi (Concertazioni, 1966) consistant uniquement en variations de timbre sur cette même note $s i^{3}$ (p. 51-52). Ailleurs, Bartolozzi mentionne, à la façon d'un record, que « 98 doigtés différents ont été trouvés au hautbois pour le $\left[s i^{3}\right] »(\mathrm{p} .14)$.

15. L'expression «mélodie de timbres » n'est pas employée par Berio mais est spontanément venue à Holliger lors de notre entretien en 2014. Elle se trouve déjà sous forme de conseil aux compositeurs sous la plume de Bartolozzi : "The best way for a composer to learn the monophonic resources of tone colour is for him to write "melodies of timbre" for each woodwind instrument $»$ (op. cit., p. 62).

16. Cette dernière est aujourd'hui considérée comme perdue.

17. Shreffler, art. cité, fig. 28, p. 109. Ce trille a été utilisé par Berio, mesure 24 de la version de la partition qu'il adresse à Holliger en mai. Voir Gartmann, op. cit., p. 61, pour le relevé des autres annotations. 


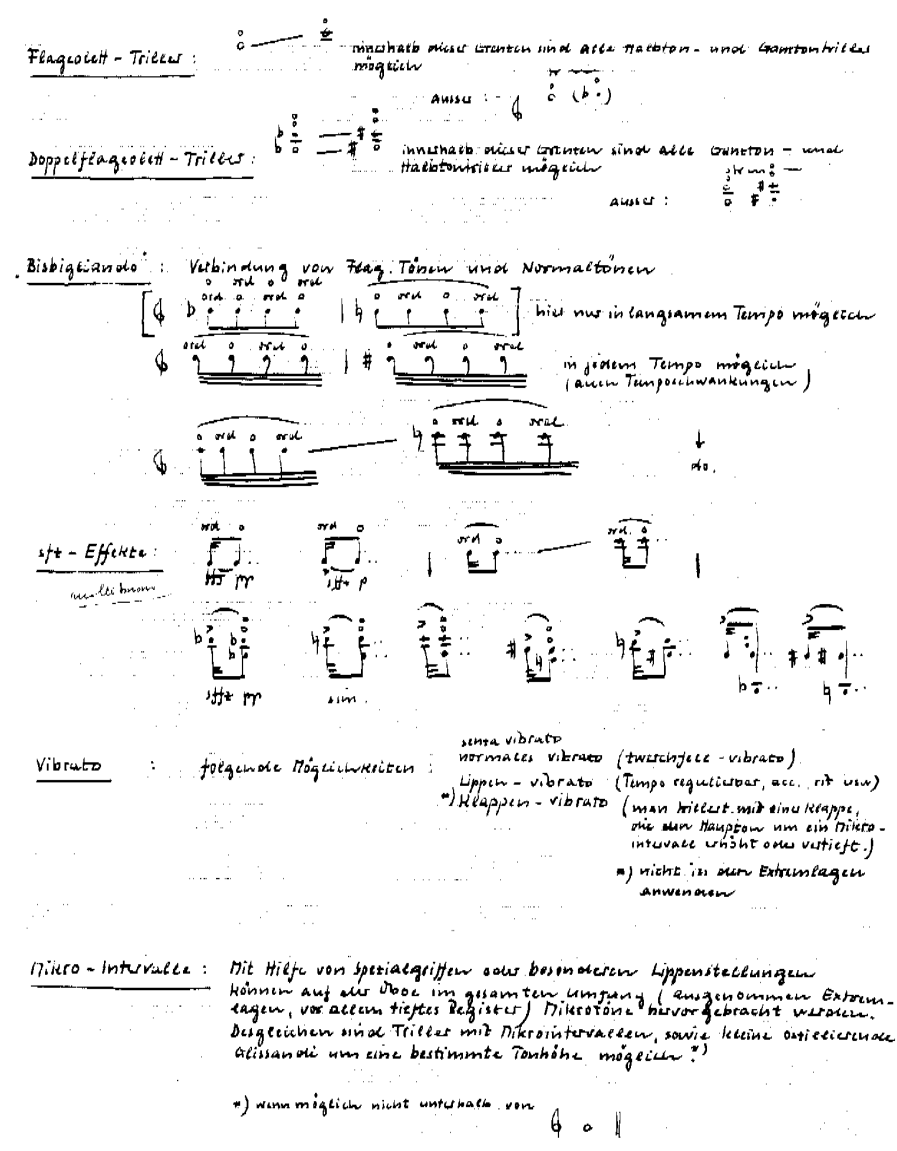

ind

Fig. 2 : Heinz Holliger, « Tabelle neuer Möglichkeiten der Oboe », p. [2] (photocopie du manuscrit avec annotation « molto buono » de Luciano Berio entre les onzième et douzième portées)

(Fondation Paul Sacher, Collection Luciano Berio)

\section{L'interprète-commanditaire, récepteur actif de l'œuvre}

La composition achevée qu'envoie Berio à Holliger en mai 1969 n'est pas la Sequenza VII telle que nous la connaissons. Son matériau, certes, est pratiquement identique : une même série de hauteurs fixes symétriquement polarisées autour d'un $s i^{3}$ incessamment varié dans ses attaques, timbres et nuances; de nombreuses formules mélodiques communes; le même usage des harmoniques doubles, trilles glissando et doubles trilles. Mais leur mise en ouvre diffère par plusieurs aspects importants, comme une structure plus aérée et continue, l'absence de sons multiphoniques et d'overblowing, l'absence de note tenue à l'arrière-plan, et surtout le fait que la Sequenza se présente ici en notation traditionnelle (mesurée et sur portée), sans aucun usage de la notation dite proportionnelle (où les distances graphiques doivent être transposées chronométri- 
quement) ni d'une grille temporelle régulière - qui sont autant de caractéristiques majeures de l'œuvre sous sa forme définitive. Présentée à Zagreb sous le titre Sequenza VII, cette première rédaction du solo sera bientôt déclarée par Berio "versione provvisoria " (comme il l'ajoute en page de titre de l'autographe sur la suggestion de sa maison d'édition), au profit d'une réécriture en profondeur par laquelle Berio la remplacera définitivement au cours de l'été suivant. Et ce n'est qu'à la demande de Holliger qu'elle paraîtra ultérieurement dans un recueil pédagogique, avec le statut de propédeutique à la Sequenza ${ }^{18}$.

Thomas Gartmann a procédé à une comparaison minutieuse des différences entre les deux versions et a documenté le processus ultérieur de gravure de la version définitive par Universal Edition ${ }^{19}$. Il n'avait malheureusement pas accès, lors de sa recherche, à la correspondance entre les musiciens. Disposer de ce chaînon manquant permet de compléter son travail de reconstruction. En particulier, la lettre de Holliger à Berio du 19 mai 1969 (incluant un post-scriptum daté du 20 mai) offre un aperçu de la phase privilégiée où l'interprète, qui est aussi le commanditaire, reçoit la partition et l'assimile.

Dans une lettre accompagnant l'envoi de la partition achevée, Berio avait commenté les numéros cerclés désignant cinq « degrés [...] de transformation par rapport à un mode conventionnel de production du son », en priant Holliger « de décider [lui]-même les doigtés plus convenables ${ }^{20} »$. L'interprète reformule cette requête et lui donne une réponse systématique dans la lettre qu'il adresse au compositeur au retour de la création de la partition à Zagreb (fig. 3a-c). Il ne cherche pas, à vrai dire, à fixer les doigtés ; comme il l'écrira ultérieurement dans l'appendice à son édition de cette première version de la Sequenza : «Pour certaines notes [...], il importera de trouver une suite de doigtés alternatifs qui permettent différents timbres sur une même hauteur. [...] Ne considérer les doigtés sur le tableau [p. 2] que comme des suggestions ; à chaque étudiant de choisir pour soi-même d'autres doigtés convenant mieux à son propre instrument ${ }^{21}$. » Holliger reconduit ainsi la délégation (du compositeur vers l'interprète) du choix des doigtés spécifiques pour les notes synonymes. En revanche, il intervient là où il a repéré des contradictions entre différents paramètres (dynamique, registre et doigtés) dont Berio ne pouvait connaître, pour chaque note donnée, les interrelations particulières liées à la facture de l'instrument : "Suivant la dynamique j'ai quelquefois interverti les chiffres de doigtés, parce que quelques doigtés ont des limites pour l'échelle de la dynamique. En plus de ça j'ai ajouté des changements de doigtés sur les $s f p[\ldots]^{22}$. » Ces corrections imposent un principe de réalité instrumentale à certaines options du compositeur qui, pour décliner sa gamme de cinq timbres, «a pensé un ordre arithmétique » qui, parfois, «ne donn[ait] rien au point de vue sonore 23 ». Le début de l'œuvre est éloquent à cet égard. La rédaction originale de Berio, inspirée par une figure de sforzando proposée par Holliger (voir supra, fig. 2), donnait à lire une variation de dynamique sur le même doigté puis trois doigtés successifs sur la même note tenue - (1), (2) et (4) - ce qui permettait d'installer le jeu de changements de degré timbral sans pour autant parcourir d'entrée de jeu la totalité de ces degrés (fig. 4a). Les degrés (3) et (5) étaient introduits dans un second temps (mes. 4 et 6). L'épreuve de la pratique rend bientôt caduque cette option. Holliger préconise d'enchaîner directement le si normal et un si sur un autre doigté, au timbre le plus différencié, puis de « revenir » vers le premier degré progressivement (fig. 4b). À la suite de cette modification puis de l'écoute par Berio (probable mais non documentée) de l'enregistrement de la création, le compositeur optera pour une troisième solution dans la version finale, réservant le doigté normal aux nuances fortes et commençant les variations timbrales par l'alternance (3) (5) (fig. 4c) qui, dans sa première rédaction, n'intervenait qu'en second.

18. Luciano Berio, Studie zu Sequenza VII, dans Pro Musica Nova. Studien zum Spielen Neuer Musik für Oboe, éd. Heinz Holliger, Cologne, Gerig, 1973, puis Wiesbaden, Breitkopf \& Härtel, 1980, p. 17-22 et Appendice, p. 1-2. Cette version de l'œuvre est cataloguée sous le titre Studie (séparément de la Sequenza VII finale) dans la Collection Berio de la Fondation Paul Sacher.

19. Thomas Gartmann, op. cit., p. 60-76, particulièrement 69-72.

20. Cité par Anne Shreffler, art. cité, p. 110.

21. Heinz Holliger, «Appendice aux "Études pour jouer la musique d'avant-garde" pour hautbois. Explication des signes et commentaires », dans Pro Musica Nova..., op. cit., p. 1.

22. Lettre de Holliger à Berio datée du 19 mai 1969. Fondation Paul Sacher, Collection Luciano Berio, correspondance.

23. Entretien avec Holliger, 4 décembre 2014. 
Heimz HoLlioen

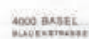

de. 14 mani 1469

the mamion Berio

comme zons mistivg wouth

que strun stygez in troyage je me whos ai pas

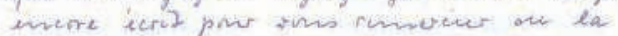
his bele Lequenga pour hawttos que jas fome sure teancomp on succis à gazreb th 35 mai. J'ar en teanconp ole plevisit en

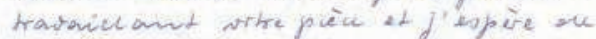
ponvoir à pen preo réahiser un que orms

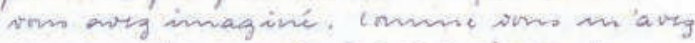
domer witre accoral je me stie permis ole yoire quelques petites retowches convenat.

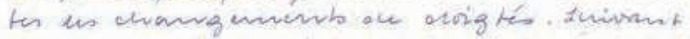

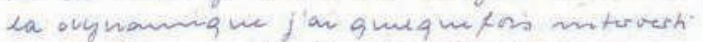

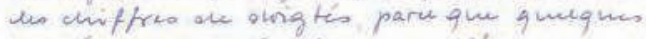

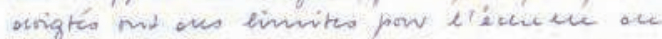
la olymanigur. in pless on ga ian agmiti ons chanigunusts oce arigtes sut us If, et at emas hangé le ownus

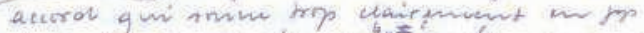

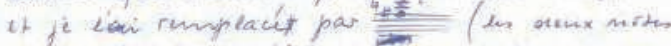

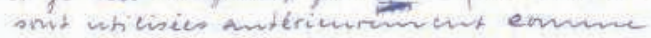

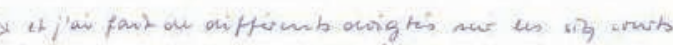

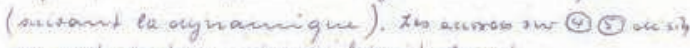

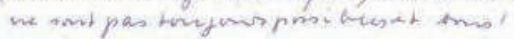

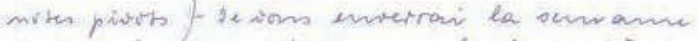
prochanin and tanace ole la piece it ta musique area lis queques hange.

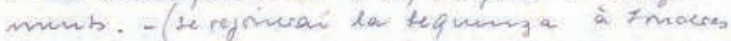

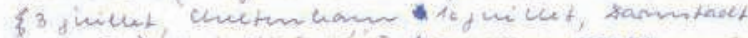

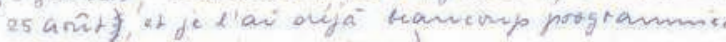
pour la prochane sasion).

quand est. ce que noms pourrims nows oost

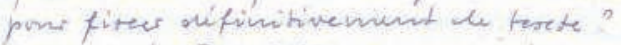

quile est wibre dotrease mianitumant?

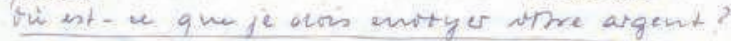

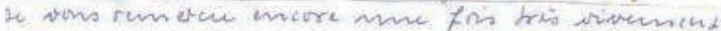

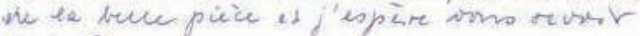
tumito

$$
\begin{aligned}
& \text { croviakmont à ins } \\
& \text { whe rewis op recife }
\end{aligned}
$$

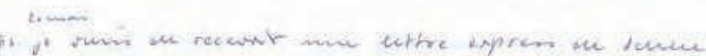

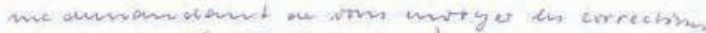

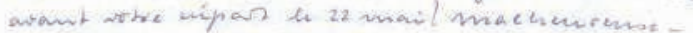

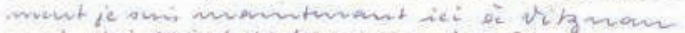

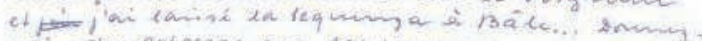

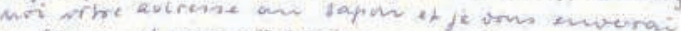

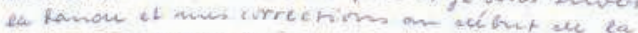
Aminame procle binse

$$
\text { ime amition } \frac{10 !}{10}
$$

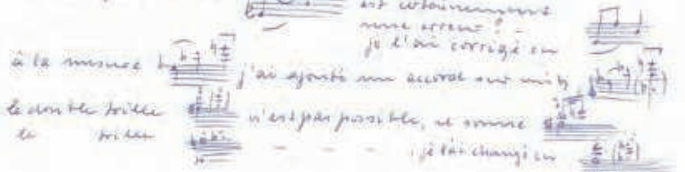

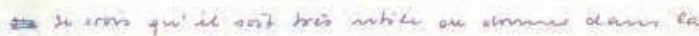

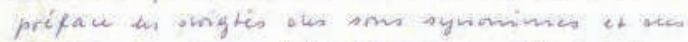

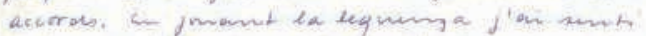

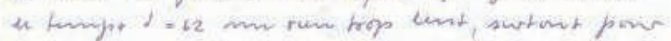

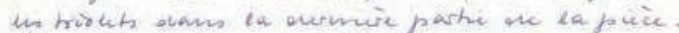

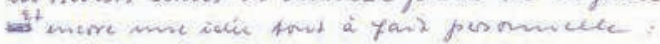

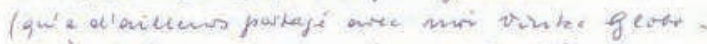

thar): He boune que le centre we la pièce une

ant pas asoez eleme, asseg agite et asosy erry

pow fustifur mone. ctoca' (triekts) calum et

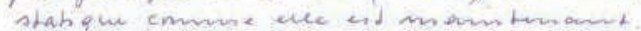

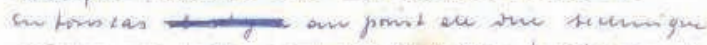

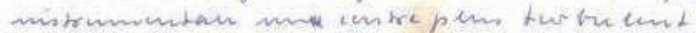

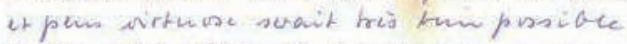

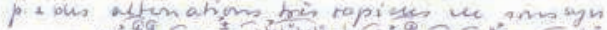

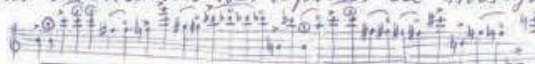

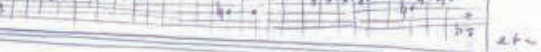

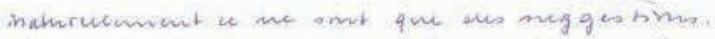

mars comme je punse que la conceptions oce eo

posthe frivale ait eté itathe mitout par rappors

enx hmitos instrimentales $f^{i}$ me sus

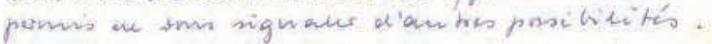

- Eeriven. moi vitre adrase it antus- mon

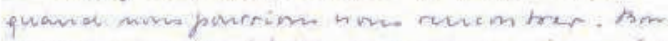

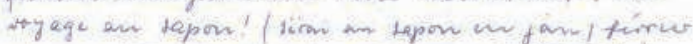

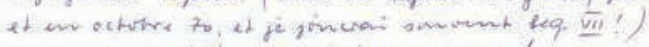

Encore ine fors muse beameonp!

Q.

Fig. 3 (a-c) : Heinz Holliger, lettre à Luciano Berio datée du 19 mai 1969 avec post-scriptum du 20 mai 1969

(Fondation Paul Sacher, Collection Luciano Berio, correspondance) 


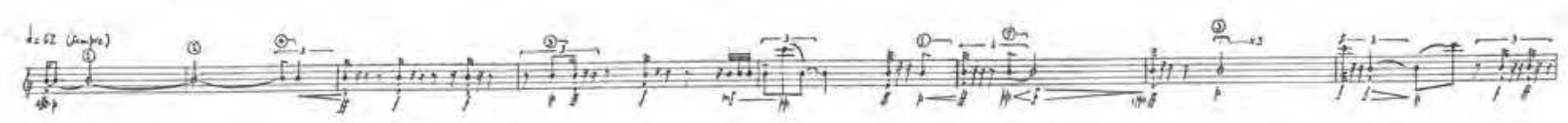

Fig. 4a : Luciano Berio, Sequenza VII, «versione provvisoria », manuscrit autographe, Fondation Paul Sacher, Collection Luciano Berio, détail (mes. 1-8). N.B. : référencement dans l'inventaire de la collection sous le titre Studie

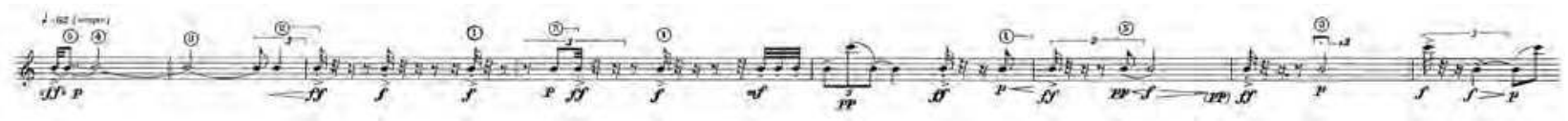

Fig. 4b : Luciano Berio, Studie zu Sequenza VII, dans Pro Musica Nova. Studien zum Spielen Neuer Musik für Oboe (Heinz Holliger, ed.), Cologne, Gerig, 1973, mes. 1-8

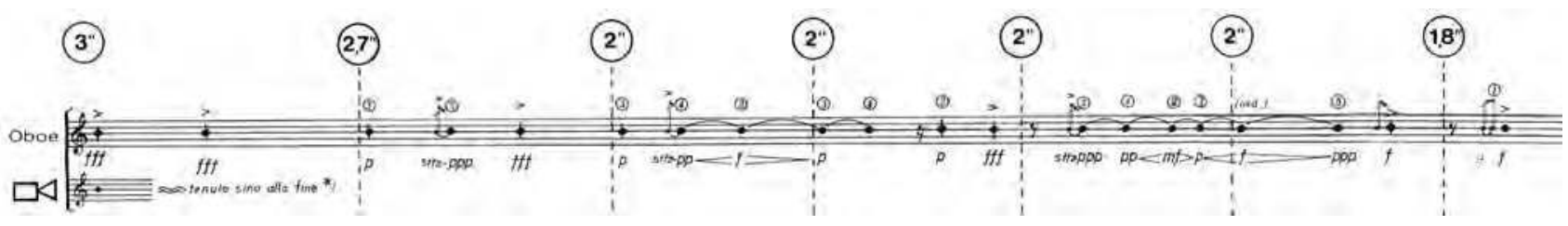

Fig. 4c : Luciano Berio, Sequenza VII, Vienne, Universal Edition, 1971, mes. 1-7

La lettre se poursuit par d'autres modifications qui, cette fois, n'affectent pas uniquement l'interrelation doigté/nuance/timbre, mais également les hauteurs. Pour pouvoir retirer l'accord final $d o \# 4-r e^{4}-d o^{5}$, qui « sonne trop clairement en $p p »$, Holliger doit faire une proposition satisfaisante non seulement au plan instrumental mais aussi au plan stylistique. Son choix engage tacitement une entente du style de la Sequenza: si la note supérieure doit être conservée, c'est certainement à la fois pour des raisons de phrasé et de hiérarchie harmonique et formelle (c'est la deuxième hauteur émise après le $s i$ au début de la pièce et elle joue un rôle de pôle par la suite). À l'inverse, fa\#t peut d'autant mieux remplacer $d o \# 4$ et rét qu'il joue un rôle polaire plus important qu'eux dans la partition. Cette vision des choses, reflétée dans la justification explicite apportée par Holliger ( « les deux notes sont utilisées antérieurement comme notes pivots $24 ! »)$, ne retient pas d'autres critères analytiques possibles tels que le nombre de hauteurs, leur écartement de registre ou leur degré de dissonance.

Quoique de nature et de quantité modestes par rapport à la partition composée par Berio, les corrections de Holliger vont donc nécessairement au-delà de l'approche strictement technique qui consisterait à accepter les doigtés les plus tortueux pour garantir un respect total de la lettre du texte reçu, quand bien même ce dernier témoignerait par endroits d'un défaut de maîtrise des possibilités de l'instrument par le compositeur. Mais la suite de la lettre - un long post-scriptum rédigé le lendemain des passages que nous avons commentés (fig. 3) - montre mieux encore la capacité qu'a eue Holliger de se tenir sur la crête entre exécution et composition pour détecter (et écarter) toute forme d'imperfection ou d'inaboutissement susceptible d'affecter ces pages virtuoses qu'il comptait jouer fréquemment (comme en témoignait déjà sa promesse à Berio dans le tout premier courrier, que nous avons déjà cité, et comme le confirme rétrospectivement l'abondance d'exécutions de l'œuvre par Holliger à travers le monde au fil des décennies). Si on lit les quatre points du post-scriptum avec la grille d'analyse qui vient d'être appliquée aux corrections de doigtés, il apparaît que les deux derniers résolvent eux aussi un problème d'exécution en tenant compte de la vraisemblance stylistique. À l'inverse, les deux premiers, respectivement une modification et un enrichissement du texte, mettent en jeu d'abord la question stylistique et en arrière-plan la pertinence instrumentale.

24. Lettre de Holliger à Berio datée du 19 mai 1969. Fondation Paul Sacher, Collection Luciano Berio, correspondance. 
Dans un feuillet non daté qui semble être la suite du post-scriptum du 20 mai, Holliger s'aventure un peu plus loin en terrain compositionnel. Il connecte une observation d'interprète (l'indication métronomique devrait être légèrement supérieure) à une considération beaucoup plus générale sur la forme de la pièce :

En jouant la Sequenza j'ai senti le tempo $\bullet=62$ un rien trop lent, surtout pour les triolets dans la dernière partie de la pièce. Et encore une idée tout à fait personnelle : (qu'a d'ailleurs partagé avec moi Vinko Globokar) : Je trouve que le centre de la pièce ne soit pas assez dense, assez agité et assez long pour justifier une „coda” (triolets) calme et statique comme elle est maintenant. En tous cas au point de vue technique instrumentale un centre plus turbulent et plus virtuose serait très bien possible.

p. e des alternations très rapides de sons syn[onymes]
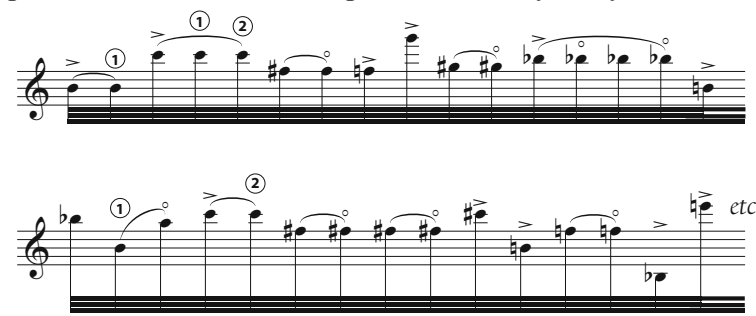

Naturellement ce ne sont que des suggestions. Mais comme je pense que la conception de la partie finale ait été établie surtout par rapport aux limites instrumentales, je me suis permis de vous signaler d'autres possibilités 25 .

Ce que pointe très précautionneusement Holliger ici, c'est un certain manque de variété et de dynamisme dans la composition. Les traits virtuoses en triples croches, dans la première version de la Sequenza, sont relativement modestes en quantité, en taille (le plus long dure moins de trois temps) et en complexité (ce sont le plus souvent des figures ascendantes ou en arabesques, ordinario et legato tout du long). Pour démontrer par l'exemple qu'il est parfaitement envisageable de marier ce même degré de vélocité avec des modes de jeux plus originaux et changeants, Holliger prend l'initiative de rédiger un passage inspiré du style de la Sequenza (mêmes notes polaires et intervalles préférentiels, emploi de plusieurs doigtés successifs et d'harmoniques pour certaines hauteurs), mais qui s'en écarte à la fois par la fragmentation du phrasé et par la longueur du trait, présenté hors de toute métrique. Tout en repoussant la frontière de la virtuosité instrumentale pré- supposée par Berio, Holliger procède donc à une extension, ou un enrichissement du style de la Sequenza ${ }^{26}$. Ces deux avancées conjointes seront parfaitement entendues par Berio au moment où il réécrira entièrement la pièce : on retrouve aux mesures 102-106 de la nouvelle version un trait semblable à celui proposé par Holliger (à l'exception des rapides alternances de liaisons et d'accents). Berio s'est réapproprié la proposition en l'intégrant à une évolution stylistique et formelle plus globale de sa Sequenza.

Revenons, pour finir, sur la question de la forme abordée dans la citation précédente - ainsi que sur d'autres aspects significatifs de l'étape de réécriture de la partition.

\section{De la relecture de Holliger à la réécriture de Berio}

Recevoir de Berio la partition qu'il lui a commandée ne signifie donc pas, pour Holliger, mettre un terme au dialogue des deux années précédentes à propos des possibilités techniques de l'instrument(iste). Au contraire, l'interprète continue d'assumer son rôle de conseiller technique, raisonnant désormais non plus sur la base des idées et questions du compositeur mais à partir de la lettre du texte qu'il a reçu. Il considère la partition avec un œil et une oreille critiques, à la lumière d'intentions compositionnelles qu'il peut d'autant mieux pressentir ou inférer qu'il a été associé à plusieurs moments décisifs de la définition du projet d'œuvre, et que son point de vue d'interprète peut à tout moment être doublé de sa propre expérience de compositeur. Il serait donc assez spécieux de vouloir déterminer si c'est le commanditaire, l'interprète, le compositeur ou l'auditeur en lui qui émet l'« idée tout à fait personnelle » que le centre de la pièce n'est pas assez « dense, [...] agité et [...] long pour justifier une "coda" $[\ldots]$ calme et statique » occupant un tiers de l'œuvre. Il veille, en tout cas, à cautionner sa remarque par l'évaluation convergente de Globokar - autre interprètecompositeur qui, étant présent au concert de Zagreb, joue le

25. Lettre de Holliger à Berio, feuillet non daté, probablement à la suite du post-scriptum à la lettre du 20 mai 1969. Fondation Paul Sacher, Collection Luciano Berio, correspondance.

26. Comme nous l'a dit Holliger plus modestement lors de notre entretien en 2014, sa suggestion de trait est avant tout « quelque chose qui va bien dans les doigts », mais pensé « à l'intérieur [du] carillon » qu'est le système harmonique de la Sequenza. 
rôle, sinon d'oreille de Berio (dont il est un proche ami), en tout cas d'auditeur aussi critique que bienveillant.

C'est, du reste, une longue lettre envoyée à Globokar par Berio depuis son lieu de vacances qui nous permet de dater la période de remise en chantier de l'œuvre quelques semaines plus tard : " Maintenant je suis en train de terminer la V partie de Sinfonia et de (re)ecrire serieusement Sequenza pour hautbois 27 . » Cette refonte permet à Berio d'intégrer davantage d'idiomatismes inspirés par le jeu de Holliger et d'aiguiser le propos de l'œuvre, avant que cette dernière ne connaisse une diffusion importante à partir du mois d'août avec sa présentation à Darmstadt et son enregistrement sur un disque Philips, sans compter sa possible intégration comme partie soliste d'une ouvre pour hautbois et cordes évoquée dès cette époque par Berio 28 . Un courrier de Holliger fin juin montre toutefois que Berio n'avait pas ouvertement annoncé une réécriture, puisque Holliger continue de s'attendre à une finalisation de la version existante :

Par le même courrier je vous envoie mon 2ème exemplaire de Sequenza VII avec les changements que j'ai fait pour Zagreb. Comme vous voyez j'ai employé les différents doigtés en fonction de la dynamique (avec aussiffp = changement de doigté etc). Mais pour établir vraiement une version définitive je crois qu'il soit indispensable que nous nous rencontrions encore une fois. Je dois être à Londres le 2 juillet soir. Je pourrais passer par Amsterdam. Est-ce que vous auriez le temps p. e. le 2 juillet après-midi ? [...]

Philips aimerait enregistrer votre pièce déjà en août. Jusqu'à cette période je devrais avoir la version définitive. - Je me réjouirais beaucoup d'avoir aussi une version avec cordes 29 !

La nouvelle version composée par Berio au début du mois d'août, si elle reconduit nombre d'aspects fondamentaux de la rédaction première, introduit aussi certaines caractéristiques que la postérité a précisément considérées comme la signature de cette œuvre.

Tout d'abord l'accompagnement du hautbois par une note tenue en arrière-fond, situation nouvelle par rapport aux six Sequenze précédentes : «Un si doit résonner tout au long de la pièce. Il est préférable que la source sonore ne soit pas visible; ce peut être un oscillateur, une clarinette, un hautbois préenregistré, ou autre $[\ldots]^{30}$. Cette décision, qui vient résoudre l'hésitation ancienne du compositeur entre un « projet hautbois-bande 31 » et un pur solo (incarné par la version provisoire), en introduisant une distinction entre figure et fond qui intensifie, de façon auditive et scénique, des propriétés préexistantes de la composition : la structuration harmonique autour du $s i$, le jeu sur les variations de couleur de cette même note, la dialectique entre continuité et discontinuité du discours.

D'autre part, Sequenza VII est désormais caractérisée par une temporalité bien spécifique : le solo suit une grille chronométrique sous-jacente (les mesures sont remplacées par des durées qui reviennent cycliquement) à l'intérieur de laquelle une certaine flexibilité locale est permise. En effet, la présentation adoptée associe librement notation proportionnelle (ou « space notation ») et notation mesurée traditionnelle. La partition ne sera pas éditée en livret mais se présente sous la forme d'un grand feuillet (plié en quatre) de 13 mesures $\times 13$ lignes, manifestant la régularité de la structure 32 .

Last but not least, la réécriture des phrases et gestes constitutifs du solo, guidée par la contrainte nouvelle de la grille temporelle, a abouti à un discours plus volubile et virtuose que celui de la première version. Non seulement le diagnostic de Berio sur sa version provisoire devait-il être proche de celui de Holliger et Globokar, mais les réponses qu'il y a apportées ont tiré parti des suggestions concrètes de son interprète pour animer davantage le discours et, corrélativement, réduire l'importance formelle de la partie finale. Brève, simple et homogène, cette dernière est désor-

27. Lettre de Berio à Globokar datée du 27 juillet 1969. Fondation Paul Sacher, Collection Vinko Globokar.

28. Ce sera Chemin IV en 1974.

29. Lettre de Holliger à Berio datée du 26 juin 1968. Fondation Paul Sacher, Collection Luciano Berio, correspondance.

30. Indication en note de bas de page de la partition (Vienne, Universal Edition, 1971).

31. Lettre de Holliger à Berio datée du 29 avril 1968. Fondation Paul Sacher, Collection Luciano Berio, correspondance.

32. L'élégante présentation de la partition sur un grand feuillet ne reflète pas la volonté de Berio (ni celle de Holliger), qui préconisait une mise en page plus aérée sur deux pages (Gartmann, op. cit., note 236, p. 71-72). Nous ne traiterons pas ici de l'étape de correction des épreuves, commentée en détail par Gartmann sur la base des archives de la maison d'édition. Indiquons seulement que Holliger y a largement contribué, accompagnant jusqu'au bout l'accouchement de l'œuvre par ses corrections, conseils et propositions, y compris en dialogue direct avec les interlocuteurs d'Universal Edition. 
mais une coda au sens plein du terme - sans les guillemets par lesquels Holliger devait encadrer ce mot lorsqu'il commentait la version provisoire.

\section{Une démarcation des rôles cadrée par le projet}

Aussi finement documenté soit-il sous certains aspects, le processus de création que nous venons de retracer ne dit absolument rien du moment classiquement étudié en génétique, celui de la sinueuse élaboration solitaire des énoncés par l'écrivant-auteur. Le texte de la Sequenza VII, Berio l'a produit en quelques jours de printemps puis d'été 1969 sans qu'aucune esquisse n'ait été conservée. Quelques indices dans notre dossier génétique, complétés par l'entretien rétrospectif avec Holliger, permettent de faire des hypothèses sur la nature de ce travail, mais cela excéderait les limites et le thème principal de cet article. Notons, en revanche, que l'analyse de la dimension collaborative du processus permet de pointer une double caractéristique du travail du compositeur : d'une part, son activité compositionnelle a été stimulée, facilitée, influencée ou encore prolongée par ses interactions avec l'interprète, du tout début à la toute fin du projet; d'autre part, la division des rôles n'a jamais été remise en cause par l'un ou par l'autre, comme le reflètent d'ailleurs bien les sources dans le contraste saisissant entre absence de traces du travail solitaire et abondance de documentation des interactions et ajustements associés à l'engagement créatif de l'interprète.

Certes, il n'y a, au premier abord, rien d'étonnant à ce qu'un compositeur et un interprète assument pleinement les rôles qui leur sont assignés par le régime occidental moderne de la division du travail musicien. Le cas présent n'a pourtant rien de simpliste. Holliger n'est aujourd'hui pas moins connu comme compositeur que comme interprète. Le développement parallèle de ces deux activités bien différentes, qui plus est à un haut niveau d'excellence, allait peut-être à contre-courant dans une époque et un milieu artistique plutôt caractérisés par des processus de spécialisation ; mais il se rencontrait aussi chez d'autres musiciens. Ainsi Globokar avait-il commencé l'apprentissage de la composition auprès de René Leibowitz après avoir fait ses premières armes comme prodige du trombone. La rencontre avec Berio, en 1964, l'encouragea à s'affranchir des approches académiques de l'écriture. Bientôt son activité de compositeur fut soutenue par des commandes ré- gulières, en parallèle de quoi il poursuivit sa carrière de tromboniste, notamment comme improvisateur. Chez Holliger, la balance entre les deux activités penchait encore nettement du côté de l'interprétation au moment de l'échange avec Berio (qui, d'après le souvenir de Holliger, n'avait pas connaissance ni même conscience de son travail de compositeur). Ce n'est qu'à partir des années soixante-dix que sa reconnaissance en tant que compositeur a pris un tour international et pérenne. Quant à Berio, son activité musicale fut loin de se limiter à la composition, en particulier dans les années soixante pendant lesquelles s'épanouit sa carrière de chef d'orchestre, notamment aux États-Unis où il enseignait et résidait alors.

Cette multiplicité des rôles s'observe encore mieux à l'échelle locale de la période 1967-1969, au cours de laquelle se déploie le projet de Sequenza VII. En effet, en parallèle de son dialogue avec Berio, Holliger mène d'autres projets, tant de compositions personnelles que de commandes à d'autres, visant à l'exploration des possibilités techniques et esthétiques du hautbois (et plus généralement des instruments à vent). En 1967-1968, il compose $h$, pour quintette à vent, qui s'appuie largement sur les sons recensés par Bruno Bartolozzi - tout particulièrement le si qui donne son titre à l'œuvre. À la réflexion solitaire de Berio sur les potentialités compositionnelles exceptionnelles de cette note au hautbois faisait donc discrètement écho une autre réflexion solitaire, parallèle, de la part de Holliger, sans que ce dernier éprouve le besoin d'interférer en cette qualité avec le processus créateur qu'il avait suscité par sa commande. La création de l'œuvre n'eut lieu que le 29 août 1969 à Darmstadt, dans le même concert que celle de Sequenza VII (version définitive). Holliger, au contraire de Berio, a certainement reçu et aimé cette dernière sur fond de sa propre exploration compositionnelle de la multiplicité des doigtés et couleurs de si possibles.

Il ne fait donc pas de doute que les deux protagonistes de la genèse de Sequenza VII ont fait bénéficier le compositeur en eux des savoirs et expériences de l'interprète, et vice versa. Cette polyphonie intérieure ne se reflète pourtant pas de manière évidente dans les sources que nous avons analysées. Dans ses remarques et suggestions à Berio, Holliger se tient parfois sur la crête entre interprétation et composition, mais il ne franchit la ligne qu'une seule fois - et en partie au nom d'un tiers. Berio, quant à lui, démarque classiquement les aspects de la partition qui concernent l'interprète (comme le choix du doigté/timbre particulier au sein d'une grada- 
tion par lui définie) de ceux qui relèvent intégralement de son autorité (à commencer par les hauteurs et le découpage temporel). En somme, c'est précisément parce qu'ils savent très bien, par leurs pratiques d'interprètes et de compositeurs, qu'une nette distinction entre ces deux rôles est souvent la meilleure garantie de leur concours harmonieux, que Holliger et Berio prennent soin de ne pas faire de pas de côté. Dès le départ, la formulation du projet esthétique définit un véritable cadre interactionnel qui détermine la place de chacun et son champ d'action. À la même époque, chez d'autres compositeurs, différents types de projets esthétiques impliquaient une participation beaucoup plus directe de l'interprète aux choix « compositionnels », qu'il s'agisse de l'agencement des parties mobiles d' une œuvre en archipel, de la définition des conventions de lecture d'une partition graphique émancipée des conventions habituelles du solfège, ou encore de la contribution égalitaire à un processus d'improvisation indifférent aux disparités d'expertise. Berio n'a pas marqué un grand intérêt pour ces paradigmes alternatifs et ses collaborations étroites avec les interprètes des Sequenze et d'autres œuvres ont rarement fait place à une transformation des fonctions conventionnelles de la notation musicale. Quant à Holliger, cohérent aujourd'hui comme hier avec cette claire répartition des rôles, il tend à minorer rétrospectivement son propre apport 33 - à l'inverse peut-être d'interprètes qui, n'occupant jamais par ailleurs le rôle du compositeur, tiendraient à signaler le caractère déterminant de leurs apports individuels aux processus créateurs auxquels ils ont pris part.

$*$

Dans une introduction aux enjeux de réalisation instrumentale de Sequenza VII, l'un des interprètes actuels de l'œuvre, Christopher Redgate, s'émerveille de l'excellence des « choix faits par Berio » en termes « d'écriture idiomatique pour le hautbois ». Il rend notamment hommage au compositeur pour la délimitation pertinente du registre utilisé et pour le $s i^{3}$ axial « qui ne pouvait pas être mieux choisi ${ }^{34}$ ». C'est, au regard des sources et de notre analyse, un contresens tant sur le travail compositionnel de Berio que sur la notion de choix (même s'il est bien entendu que Redgate se place, en l'occurrence, dans une perspective de recherche en interprétation et non en musicologie).

Ces louanges mal placées montrent bien que l'a priori de l'auteur omnipotent et omni-intentionnel a la vie dure, même (surtout ?) dans le domaine de la musique contemporaine, où il est pourtant bien connu que les interprètes mettent volontiers leur esprit de recherche instrumentale au service de l'innovation compositionnelle. Prendre au sérieux ce caractère collaboratif dans un corpus comme les Sequenze de Berio, n'implique certainement pas de transférer la propriété d'auteur mécaniquement aux interprètes, mais d'identifier les différentes formes de savoirs, méthodes, idées, matériaux conjointement mises en jeu par les différents agents du processus créateur, en sachant que ces derniers ne mobilisent pas les mêmes compétences en tous lieux et en toutes circonstances ${ }^{35}$. Dès lors, comprendre la logique interne de la genèse, c'est aussi montrer comment cette dernière est un lieu performatif d'individuation des rôles pour ses participants, ainsi que de réaffirmation ou de redéfinition des contours mêmes de l'œuvre.

Cette étude a été réalisée dans le cadre du projet «Geste musical : modèles et expériences » subventionné par l'Agence nationale de la recherche (2012-2016), associant l'équipe Analyse des pratiques musicales (Ircam) et le Centre transdisciplinaire d'épistémologie de la littérature et des arts (Université de Nice-Sophia Antipolis).

33. «[Berio] avait une idée précise. Il a dit qu'il voulait qu'une note soit pédale, et que tout soit symétrique par rapport à cet axe. Il m'a demandé quel son serait le meilleur. Mais de son côté il avait déjà pratiquement toutes les idées. Et il s'est servi seulement de colorations de ces sons fixes par des doubles trilles, des doubles harmoniques, des flatterzunge, ou même la combinaison de toutes les possibilités. Mais c'est une pièce qui n'avait pas tellement besoin de moi, même s'il s'est inspiré de ma façon de jouer et de ce que je lui ai montré » (entretien avec Holliger, 4 décembre 2014, transcription révisée).

34. Christopher Redgate, « Performing Sequenza VII », Contemporary Music Review, vol. 26, n 2, 2007, p. 219-230 (ici p. 219).

35. Ce travail a récemment été entrepris à propos de Cathy Berberian, épouse de Berio à l'époque de la Sequenza III et de ses précédentes œuvres vocales (voir notamment Kate Meehan, Not Just a Pretty Voice. Cathy Berberian as Collaborator, Composer and Creator, PhD. Diss., Washington University in St. Louis, 2011). Pour l'amorce d'une réflexion plus générale sur la notion de créativité distribuée appliquée à la musique contemporaine, voir Nicolas Donin, «Domesticating Gesture: the Collaborative Creative Process of Florence Baschet's StreicherKreis for "augmented" string quartet (2006-2008) », dir. Eric Clarke et Mark Doffman, Creativity, Improvisation and Collaboration: Perspectives on the Performance of Contemporary Music, New York, Oxford University Press, à paraître en 2016. 
NiCOLAS Donin est musicologue à l'Institut de recherche et de coordination acoustique/musique, où il dirige l'équipe Analyse des pratiques musicales (Ircam-CNRS-UPMC). Il est l'auteur d'études sur les processus de création musicale, l'histoire des pratiques d'écoute et d'analyse musicale depuis la fin du XIXe siècle, et l'analyse musicale d'interprétation, publiées à la fois dans le champ musicologique et dans celui des sciences humaines. Il est coauteur de courts-métrages, de DVD-Roms et de publications en ligne issus de ces recherches.

Nicolas.Donin@ircam.fr

\section{Engagements créatifs. Luciano Berio, Heinz Holliger et la genèse de la Sequenza VII pour hautbois}

Le musicien interprète n'est-il que l'humble courroie de transmission d'une expression sonore déterminée une fois pour toutes, dans le passé, par le travail solitaire de confection de la partition par le compositeur? La musicologie a répondu fermement par la négative depuis la fin du $\mathrm{xx}^{\mathrm{e}}$ siècle en réévaluant considérablement les formes de créativité propres aux interprètes et en cassant l'idée que la partition serait garante d'un sens non ambigu et éternellement stable. Mais quelle est la contribution créative de l'interprète lorsque celui-ci est directement impliqué dans le processus de création ? La genèse de la Sequenza VII pour hautbois (1969) de Luciano Berio permet de donner quelques réponses, car sa dimension collaborative est bien documentée, principalement par la correspondance entre Berio et le hautboïste Heinz Holliger (conservée à la Fondation Paul Sacher, Bâle). Holliger a été l'initiateur du projet d'œuvre, son commanditaire, son conseiller (pour l'utilisation des spécificités techniques de son instrument), son premier lecteur et interprète, son préfacier, son relecteur (jusqu'à l'étape des épreuves de la version imprimée par Universal Edition), et son principal interprète au concert comme au disque. Les modalités de cette collaboration et leurs incidences sur notre compréhension de l'œuvre (et plus généralement des processus de création musicale) sont discutées sur la base des différentes archives disponibles ainsi que d'un entretien inédit avec l'interprète.

Is the music performer only the humble transmission belt of a sound expression determined once and for all, in the past, by the composer's solitary writing of the score? Musicology has firmly answered this question negatively since the end of the $20^{\text {th }}$ century by largely reassessing the forms of creativity inherent to performers and rejection the notion that the score could guarantee a clear and permanent meaning. But what is the creative contribution of the performer when he/she is directly involved in the creative process itself? The genesis of Sequenza VII for oboe (1969) by Luciano Berio provides a few answers, because its collaborative aspect is thoroughly documented, mainly by the correspondence between Berio and the oboist Heinz Holliger (held at the Paul Sacher Foundation in Basel). Holliger was the instigator of the project of the work, its sponsor, its advisor (for the use of the mechanical specificities of his instrument), his first reader and performer, the author of the preface, and proofreader (up to the proofing stage of the version printed by Universal Edition) and principal performer in the concert and on the record. The modalities of this collaboration and their incidences on our comprehension of the work (and more generally the processes of musical creation) are discussed on the grounds of the different available archives, as well as an unpublished conversation with the oboist.
Ist der interpretierende Musiker nur das bescheidene Förderband der Übertragung eines akustischen Ausdrucks, der ein für allemal in der Vergangenheit festgelegt wurde, und zwar durch die einsame Arbeit des Komponisten, der die Partitur erstellt hat? Die Musikwissenschaft hat diese Frage seit dem Ende des 20. Jahrhunderts klar verneint, indem sie auf eine beachtliche Weise jenen Formen der Kreativität neue Beachtung schenkte, welche den Interpreten der Musik eigen sind, und indem sie die Vorstellung widerlegte, wonach die Partitur der Garant eines unzweideutigen und ewig gleichbleibenden Sinnes sei. Aber was ist nun der kreative Beitrag des Interpreten, wenn er direkt in den Entstehungsprozess einer Komposition involviert ist? Die Entstehung der Sequenza VII für Oboe (1969) von Luciano Berio vermag uns einige Antworten auf diese Frage zu geben, denn in diesem Falle ist die kooperative Dimension der Komposition gut dokumentiert, vor allem durch die Korrespondenz zwischen Berio und dem Oboisten Heinz Holliger (archiviert in der Paul Sacher Stiftung, Basel). Holliger war der Initiator des Kunstwerks, der Auftraggeber, und Berater (was den Umgang mit technischen Besonderheiten seines Instruments betrifft), er war weiters sein erster Leser und Interpret, der Autor des Vorwortes, erneuter Leser (bis hin zur Phase der Druckfahnen, hergestellt von der Universal Edition), schließlich war er auch sein erster Interpret sowohl im Konzertsaal als auch bei der Platteneinspielung. Die Modalitäten dieser Zusammenarbeit und ihre Wirkungen auf unser Werkverständnis (und allgemeiner auf das Verständnis des Entstehungsprozesses einer Komposition) werden im vorliegenden Artikel erörtert, und zwar auf der Basis verschiedener Archivmaterialien wie auch eines unveröffentlichten Interviews mit dem Interpreten.

I 1 musicista interprete è soltanto l'umile cinghia di trasmissione di un'espressione sonora stabilita definitivamente, nel passato, dal lavoro solitario del compositore che realizza la partitura? Già dalla fine del ventesimo secolo, la musicologia ha fermamente risposto in modo negativo alla domanda, rivalutando notevolmente le forme di creatività proprie agli interpreti e rigettando l'idea che la partitura sarebbe garante di un senso non ambiguo ed eternamente stabile. Ma qual è il contributo dell'interprete alla creazione quando questi è coinvolto direttamente nel processo creativo? La genesi di Sequenza VII per oboe (1969) di Luciano Berio permette di dare qualche risposta, poiché la sua dimensione collaborativa è ben documentata, principalmente dalla corrispondenza tra Berio e l'oboista Heinz Holliger (conservata alla Fondazione Paul Sacher di Basilea). Holliger è stato l'iniziatore del progetto, il finanziatore, il consigliere (sull'uso di alcune particolarità tecniche del suo strumento), il primo lettore e interprete, il prefatore, il revisore (fino alla fase delle bozze della versione stampata dalla Universal) 
ed il principale interprete, sia in concerto sia sul disco. Le modalità di questa collaborazione e la loro incidenza sulla nostra comprensione dell'opera (e, più in generale, dei processi di creazione musicale) vengono esaminati sulla base dei diversi archivi disponibili e di un'intervista inedita con l'interprete.

Será o intérprete musical uma simples correia de transmissão da expressão sonora que ficou determinada de uma vez por todas, no passado, pelo trabalho solitário do compositor, no confeccionar da partitura? A musicologia responde firmemente que não desde o final do século $\mathrm{XX}$, ao valorizar as formas de criatividade próprias dos intérpretes e recusando a ideia de que a partitura é garante de um sentido sem ambiguidade e eternamente estável. Mas como se manifesta o contributo criativo do intérprete, quando ele se acha diretamente implicado no processo de criação? A génese da Sequenza VII para oboé (1969), de Luciano Berio, fornece algumas respostas, pois a sua dimensão colaborativa está bem documentada pela correspondência entre Berio e o oboísta Heinz Holliger (preservada na Fundação Paul Sacher, Basileia). Holliger esteve na origem do projeto do trabalho, que encomendou ; foi ainda consultor para especificidades técnicas do seu instrumento, seu primeiro leitor e intérprete, prefaciador, revisor (até à fase das provas da versão impressa por Universal Edition) e seu principal executante, tanto em concerto como em disco. As modalidades desta cooperação e as suas implicações para a nossa compreensão da obra (e em geral dos processos de criação musical) são discutidas com base nos vários arquivos disponíveis e em uma entrevista inédita com o intérprete.
¿E1 músico intérprete no es más que una modesta correa de transmisión de una expresión sonora determinada de una vez para siempre, en el pasado, por el trabajo solitario de confección de la partitura por parte del compositor? La musicología ha respondido a este interrogante de manera terminantemente negativa, a partir de finales del siglo XX, reevaluando considerablemente las formas de creatividad propias de los intérpretes y dinamitando la idea de que la partitura representaría la garantía de un sentido no ambiguo y eternamente estable. ¿Pero cuál es la contribución creativa del intérprete cuando se encuentra directamente implicado en el proceso de creación? La génesis de la Sequenza VII para oboe (1969) de Luciano Berio permite formular algunas respuestas, ya que su dimensión colaborativa está bien documentada, principalmente por la correspondencia entre Berio y el oboísta Heinz Holliger (conservada en la Fundación Paul Sacher, Basilea). Holliger ha sido el iniciador del proyecto de la obra, su patrocinador, su consejero (en lo que respecta la utilización de las especificidades técnicas de su instrumento), su primer lector e intérprete, su prologuista, su relector (hasta la etapa de las pruebas de la versión impresa por Universal Edition), y su principal intérprete tanto en conciertos como en disco. Las modalidades de esta colaboración y sus incidencias en nuestra comprensión de la obra (y, más generalmente, de los procesos de creación musical) son considerados sobre la base de los distintos archivos disponibles y de una entrevista inédita con el intérprete. 